\title{
PD-1 blockade inhibits osteoclast formation and murine bone cancer pain
}

\author{
Kaiyuan Wang, ${ }^{1}$ Yun Gu, ${ }^{1}$ Yihan Liao, ${ }^{2,3}$ Sangsu Bang, ${ }^{1}$ Christopher R. Donnelly, ${ }^{1}$ Ouyang Chen, ${ }^{1}$ Xueshu Tao, ${ }^{1}$ Anthony J. Mirando, ${ }^{3}$ \\ Matthew J. Hilton, ${ }^{3,4}$ and Ru-Rong ji, $\mathbf{i}^{1,45}$ \\ 'Center for Translational Pain Medicine, Department of Anesthesiology, ${ }^{2}$ Department of Pharmacology and Cancer Biology, ${ }^{3}$ Department of Orthopedic Surgery, ${ }^{4}$ Department of Cell Biology, and ${ }^{5}$ Department \\ of Neurobiology, Duke University Medical Center, Durham, North Carolina, USA.
}

Emerging immune therapy, such as with the anti-programmed cell death-1 (anti-PD-1) monoclonal antibody nivolumab, has shown efficacy in tumor suppression. Patients with terminal cancer suffer from cancer pain as a result of bone metastasis and bone destruction, but how PD-1 blockade affects bone cancer pain remains unknown. Here, we report that mice lacking Pdcd1 $\left(P d 1^{-/-}\right)$demonstrated remarkable protection against bone destruction induced by femoral inoculation of Lewis lung cancer cells. Compared with WT mice, $P d 1^{1-}$ mice exhibited increased baseline pain sensitivity, but the development of bone cancer pain was compromised in $P d 1^{-/-}$mice. Consistently, these beneficial effects in $P d 1^{-/-}$mice were recapitulated by repeated i.v. applications of nivolumab in WT mice, even though nivolumab initially increased mechanical and thermal pain. Notably, PD-1 deficiency or nivolumab treatment inhibited osteoclastogenesis without altering tumor burden. PD-L1 and CCL2 are upregulated within the local tumor microenvironment, and PD-L1 promoted RANKL-induced osteoclastogenesis through JNK activation and CCL2 secretion. Bone cancer upregulated CCR2 in primary sensory neurons, and CCR2 antagonism effectively reduced bone cancer pain. Our findings suggest that, despite a transient increase in pain sensitivity following each treatment, anti-PD-1 immunotherapy could produce long-term benefits in preventing bone destruction and alleviating bone cancer pain by suppressing osteoclastogenesis.

\section{Introduction}

Cancer pain is experienced by $70 \%$ of patients with advanced stage cancer; more than $80 \%$ of this pain is attributed to metastatic cancer-induced bone pain (1-3). Generally, bone metastasis from late-stage breast, lung, thyroid, bladder, and many other cancers may form osteolytic bone lesions, leading to pathological fractures and marked bone cancer pain (4). Cancer pain substantially aggravates distressed emotion, impairs mobility and social functioning, and reduces quality of life, leading to increased morbidity and mortality (5-7). Mechanisms of bone cancer pain are complex, including both nociceptive and neuropathic components involving peripheral, central, and glial modulations (8-10) as well as overactivation of osteoclasts (11). Current treatments for cancer pain, including opioids, radiotherapy, nerve block, and bisphosphonates, have limited analgesic efficacy (12, 13). Over $50 \%$ of patients with advanced or metastatic cancer could not achieve acceptable pain relief (3), in part due to lack of deeper insights into molecular mechanisms of bone destruction and bone pain.

Authorship note: KW and YG share first authorship. RRJ and KW share senior authorship.

Conflict of interest: RRJ is a consultant of Boston Scientific and received a research grant from the company. He also serves on the board of directors of Ascletis Pharma. RRJ and KW also filed a patent, Methods and kits for treating pain $(16 / 612,909)$, in association with Duke University.

Copyright: (c) 2020, American Society for Clinical Investigation.

Submitted: September 7, 2019; Accepted: March 24, 2020; Published: June 2, 2020. Reference information: J Clin Invest. 2020;130(7):3603-3620.

https://doi.org/10.1172/JCI133334.
A revolutionary approach in cancer immunotherapy targets the immune checkpoint inhibitors, including programmed cell death-1 (PD-1). Interaction of PD-1 receptor with its ligands, PD-L1 or PD-L2, modulates immune tolerance and protects tissues from autoimmune attack (14). However, PD-L1 expressed on tumor cells also suppresses cytotoxic $\mathrm{T}$ cell activity, allowing tumor escape from immune surveillance (15). Anti-PD-1 or antiPD-L1 treatment has shown success in treating cancers, such as melanoma, lung cancer, and head and neck cancers (16-18). However, it remains unclear whether PD-1 blockade affects primary or metastatic bone cancer.

We recently demonstrated that the functional PD-1 receptor is expressed by primary sensory neurons and that activation of PD-1 by PD-L1 can suppress neuronal excitability and pain (19). In a mouse model of skin cancer, PD-L1 produced by melanoma cells masked cancer pain, while nivolumab, a clinically used human anti-PD-1 monoclonal antibody (Opdivo), could unmask cancer pain by inducing mechanical allodynia and spontaneous pain (19). As terminal cancer pain is largely derived from bone (20), it is of great importance to investigate how nivolumab affects bone destruction and bone pain after tumor progression within the BM environment. Notably, checkpoint pathways were implicated in the formation of osteoclasts. For example, the CD200/CD200R axis, which plays an inhibitory role in $\mathrm{T}$ cell response $(21,22)$, appears to modulate differentiation of osteoclasts and bone mass (23). Moreover, PD-1 deficiency leads to a reduction of osteoclastogenesis without altering the number of osteoblasts (24).

In this study, we induced bone cancer pain in WT mice and $P d c d 1$-deficient $\left(P d 1^{-/-}\right)$mice following inoculation of Lewis lung 
cancer cells (LLC) into the intramedullary canal of femurs. We found that cancer-induced bone destruction and bone pain were protected in KO mice compared with in WT mice. Injection of nivolumab i.v. provoked a rapid but transient increase in mechanical and thermal pain sensitivity. However, repeated nivolumab injections produced sustained beneficial effects for relieving cancer pain and protecting bone destruction. Mechanistically, PD-L1 promoted low-dose RANKL-induced osteoclastogenesis through JNK activation and chemokine C-C motif ligand 2 (CCL2) secretion, and nivolumab treatment blocked the osteoclast formation in vitro and in vivo in mice with bone cancer.

\section{Results}

Pd1 deletion protects against bone destruction during progression of bone cancer. We induced bone cancer in WT and $\mathrm{Pd1^{-/ }}$ mice through femoral inoculation of LLC $\left(2 \times 10^{5}\right.$ cells in $\left.4 \mu \mathrm{l}\right)$. Tumor-induced osteolytic bone destruction was continuously evaluated and scored on a scale of 1 to 5 (25) using X-ray radiographs (Figure 1, A and B). In WT mice, bone destruction developed on day 8 (score of $2.8 \pm 0.3$ ) and peaked on day 15 (score of $4.5 \pm 0.17$; Figure 1, B and C). Due to deterioration of health conditions in late stages of tumor progression, we euthanized animals within 17 days. Loss of $P d 1$ resulted in an improvement in bone destruction on days 8, 11, and 15 after LLC inoculation (Figure 1, B and C). Furthermore, x-ray scans on day 15 after LLC inoculation revealed bone fractures in $40 \%$ of WT mice (4 out of 10 mice). Strikingly, none of the $P d 1^{-/-}$mice (0 out of 10 mice) suffered bone fractures (Figure 1D).

To achieve morphological structures of bone, we also conducted micro-CT analysis with $3 \mathrm{D}$ reconstruction ex vivo on the distal part of femurs from naive mice and tumor-bearing WT and KO mice. No differences in bone histomorphometric parameters, including the ratio of trabecular bone volume to total volume (BV/ TV) and connectivity density (Conn.D), were found between naive WT and $P d 1^{-/-}$mice (Figure 1, E and F). On day 8 after tumor inoculation, WT mice exhibited marked bone destruction, as revealed by sharp decreases in BV/TV and Conn.D (Figure 1, E and F). However, these parameter changes were reversed in $\mathrm{Pd1^{-/ }}$ mice (Figure 1, E and F). Thus, bone destruction is markedly protected in $\mathrm{Pd1}^{-/-}$mice during the development of metastatic bone cancer.

Pd1 deletion increases baseline pain, but decreases cancer-induced bone pain. Bone cancer pain usually develops along with aggravated bone destruction (6). We compared cancer-induced bone pain in both WT and $P d 1^{-/-}$mice. Behavior tests of cancer pain for assessing mechanical and thermal sensitivity were performed on days $0,7,10$, and 14 after LLC inoculation (Figure 2A). On day 0 before tumor inoculation, $P d 1^{-/-}$mice exhibited increased baseline pain sensitivity compared with WT mice, showing reduction in paw-withdrawal threshold and increase in paw-withdrawal frequency in von Frey tests (Figure 2B) as well as reduction of knee-withdrawal threshold (Figure 2C) using pressure application measurement (PAM). Hargreaves and acetone tests did not show changes in baseline heat and cold sensitivity in $\mathrm{KO}$ mice (Figure $2, \mathrm{D}$ and $\mathrm{E}$ ). These data are in agreement with our previous report that $P d 1$-deficient mice have increased pain sensitivity (19).

On day 7 after tumor inoculation, WT mice developed mechanical allodynia in hind paws (Figure 2B), mechanical hyper- algesia in knees (Figure 2C), and heat hyperalgesia and cold allodynia in hind paws (Figure 2, D and E). These signs of cancer pain were maintained on day 10 and day 14 in WT mice (Figure 2, B-E). In $\mathrm{Pd1}^{-/-}$mice, cancer-induced mechanical allodynia (threshold only), mechanical hyperalgesia, heat hyperalgesia, and cold allodynia were all attenuated on day 7, but not on day 10 and day 14 (Figure 2, B-E). Notably, mechanical allodynia, measured by withdrawal frequency, was significantly reduced in $\mathrm{KO}$ mice on day 10 and day 14 (Figure 2B), suggesting a sustained improvement in mechanical pain after loss of PD-1.

We also compared sex differences in bone cancer pain in WT and KO mice. We observed a protective effect of $P d 1$ deletion on cancer pain and bone destruction in both male and female mice (Supplemental Figure 1; supplemental material available online with this article; https://doi.org/10.1172/JCI133334DS1). Specifically, WT female mice developed robust cancer pain on days 7, 10, and 14 (Supplemental Figure 2, A-D) and also exhibited greater mechanical pain than males at some time points (Supplemental Figure 2, A and B). LLC-induced heat pain, cold pain, and bone destruction were indistinguishable between sexes (Supplemental Figure 2, C-E). In KO mice, bone cancer pain and bone destruction were comparable in both sexes (Supplemental Figure 2, F, G, I, and $\mathrm{J}$ ), except for cold allodynia (Supplemental Figure $2 \mathrm{H}$ ).

Nivolumab protects against bone destruction after LLC inoculation. To circumvent developmental modulation in Pd1-deficient mice, we blocked the function of PD-1 in adult WT mice using nivolumab, as this human antibody also binds mouse PD-1 and specifically modulates neuronal activity and pain in mice (19, 26). Nivolumab or its isotype control antibody (human IgG4) was i.v. $(10 \mathrm{mg} / \mathrm{kg})$ administered every 3 to 4 days, beginning on the day of LLC inoculation (Figure 3A). Radiographs revealed that nivolumab treatment attenuated bone destruction on days 8 and 11 compared with human IgG4 (Figure 3, B and C). MicroCT scan on day 8 showed that nivolumab increased trabecular $\mathrm{BV}$ and density in the distal part of the ipsilateral femora (Figure 3, E and F). Notably, nivolumab treatment in tumor-free naive mice had no effects on the bone microstructure (see below). On day 15 , radiography revealed bone fractures in $29.4 \%$ of control IgG-treated mice ( 5 out of 17 mice). In sharp contrast, none of the nivolumab-treated mice (0 out of 19 mice) had bone fractures (Figure 3D). Subgroup analysis revealed that the bone protection by nivolumab was evident in both male and female mice (Supplemental Figure 3, A and F) and that there was no obvious sex difference in bone destruction in mice after immune therapy (Supplemental Figure 4, A and F).

As a human antibody, nivolumab also shows crossreactivity in mouse tissues, and specific binding to nivolumab was shown in dorsal root ganglion (DRG) neurons and sciatic nerve fibers in WT, but not $\mathrm{Pd1}^{-/-}$, mice (19). We examined the distribution of nivolumab in cancer-bearing C57BL/6 mice by using Vivo 680 to label nivolumab. After a single injection of nivolumab $(10 \mathrm{mg} / \mathrm{kg}$, i.v.) on day 8 after LLC inoculation, we tested the accumulation of Vivo 680-tagged nivolumab in ipsilateral and contralateral femur at 3 hours, 1 day, 2 days, and 3 days. As compared with the contralateral side, we found higher nivolumab accumulation on the ipsilateral femur on day 1 and day 2 (Supplemental Figure 5, A-C) revealed by the increased fluorescence labeling. Nivolumab was 
A

\begin{tabular}{|c|c|c|c|}
\hline $\mathrm{BL} \quad 0$ & $\underset{1}{8 d}$ & $11 \mathrm{~d}$ & $15 d$ \\
\hline adiography & $\begin{array}{c}\uparrow \\
\text { Radiography }\end{array}$ & $\begin{array}{c}\uparrow \\
\text { Radiography }\end{array}$ & $\stackrel{\uparrow}{\text { Radiography }}$ \\
\hline
\end{tabular}

B
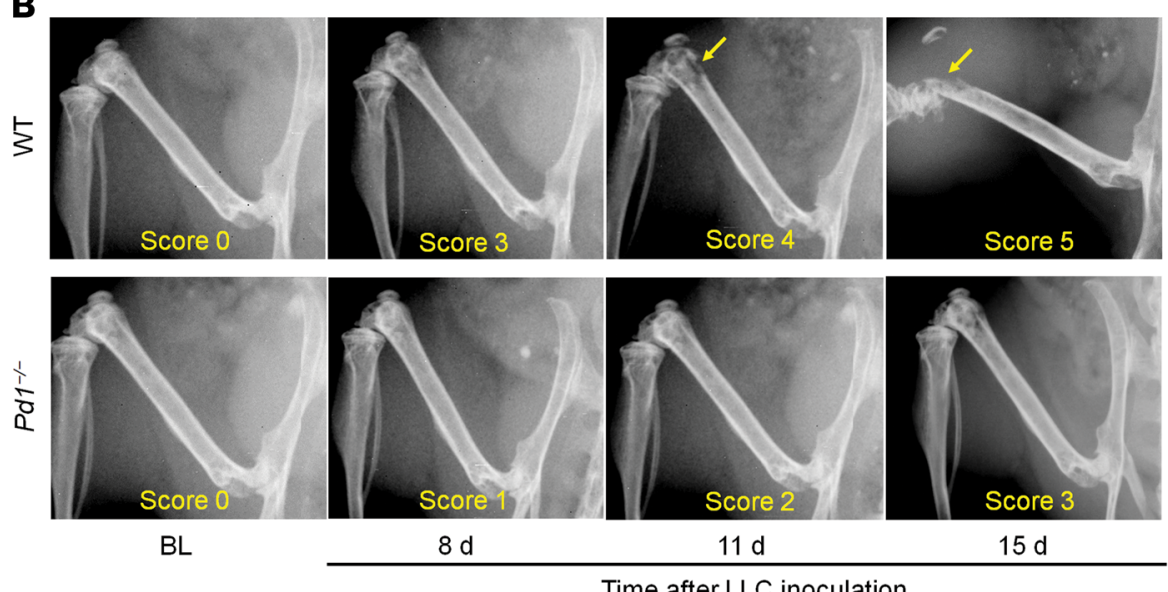

E


Tumor bearing mice ( $8 \mathrm{~d}$ after LLC inoculation)
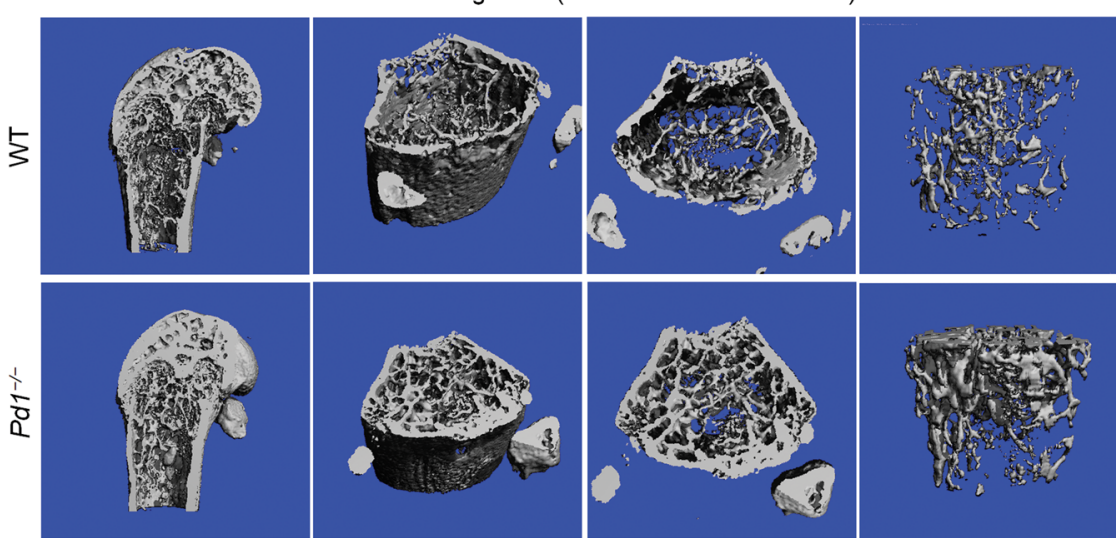
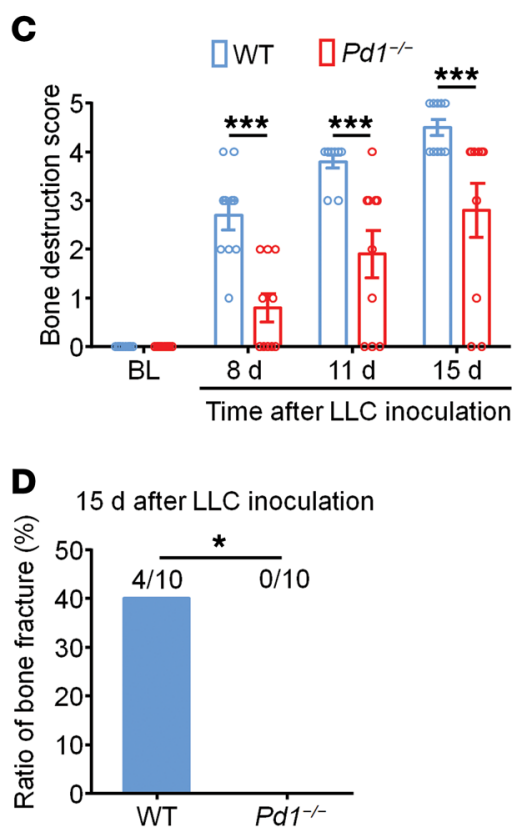

$\mathbf{F}$
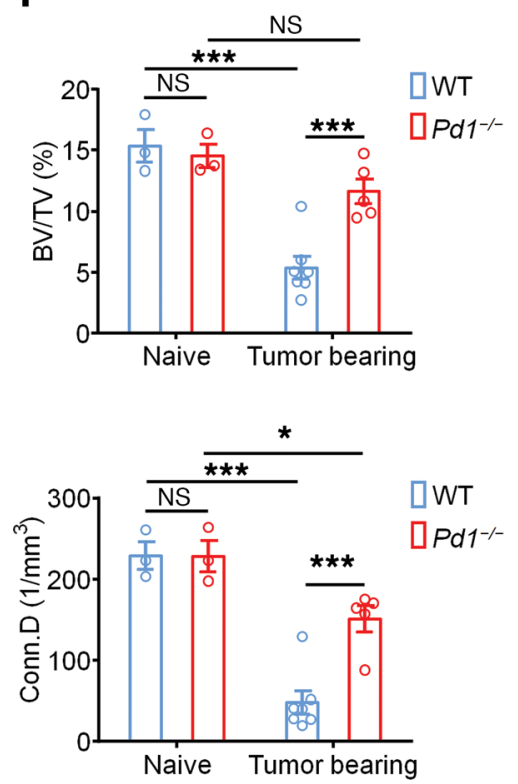

Figure 1. Protection of cancer-induced bone destruction and fracture in tumor-bearing Pd1 ${ }^{-/}$mice. (A) Experimental diagram showing LLC inoculation and radiography. (B) Radiographs of tumor-bearing femora from WT mice and Pd1 1 - mice on days 8, 11, and 15 after LLC inoculation. Bone destruction scores are indicated in photographs, and arrows indicate bone lesions with scores over 3. (C) Quantification of bone destruction scores ( $n=10$ mice). (D) Ratio of bone fracture on day 15 after tumor inoculation between WT mice and Pd1 1 - mice $(n=10$ mice). (E) Representative micro-CT images showing bone microstructure in the distal part of femora from naive WT and KO mice and tumor-bearing WT and KO mice 8 days after LLC inoculation. Note reduction of medullary bone and increased lesions in cortical bone in WT mouse after tumor implantation. (F) Quantification for $\mathbf{E}$ showing BV/TV and Conn.D in naive WT and KO mice with and without tumor ( $n=3-7$ male mice). Data are represented as mean \pm SEM. ${ }^{*} P<0.05$; ${ }^{* * *} P<0.001$, repeated measures 2 -way ANOVA with Bonferroni's post hoc test (C), Fisher's exact test (D), and 2-way ANOVA with Bonferroni's post hoc test (F). BL, baseline. 
A



B
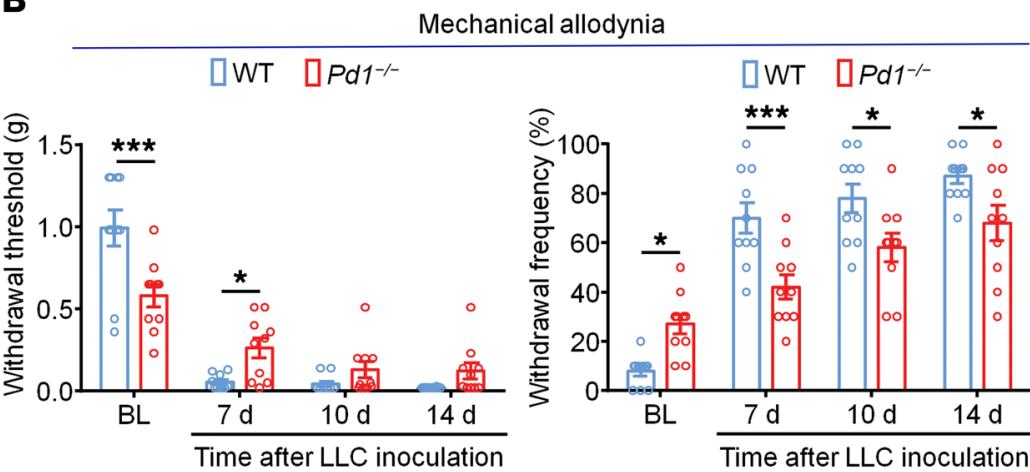

C

Knee hyperalgesia



D

E

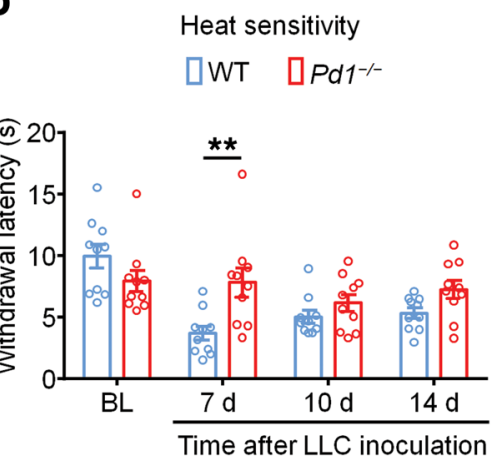

Cold allodynia

पWT पPd1/-

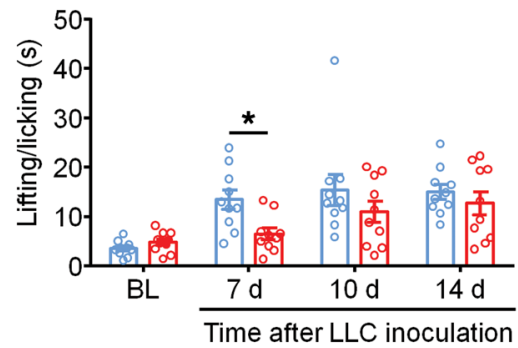

Figure 2. Reduction of persistent bone cancer pain in tumor-bearing $\boldsymbol{P d 1}^{-/-}$mice. (A) Experimental diagram for behavioral tests. (B) von Frey test of withdrawal threshold (left) and frequency (right) in WT and $P d 1^{-1-}$ mice. Note that $P d 1^{-1-}$ mice exhibit increased basal mechanical sensitivity, but reduced mechanical allodynia, after tumor inoculation ( $n=10$ mice). (C) PAM showing knee hyperalgesia in WT and Pd1/- mice $(n=10$ mice). (D and E) Hargreaves and acetone tests showing heat hyperalgesia (D) and cold allodynia (E) in WT and Pd1 1/- mice ( $n=10$ mice). Data are represented as mean \pm SEM. WT versus KO. ${ }^{*} P<0.05 ;{ }^{* *} P<0.01 ;{ }^{* * *} P<0.001$, repeated measures 2 -way ANOVA with Bonferroni's post hoc test.

also detected in serum, with a half-life of 3.36 days after i.v. injection (Supplemental Figure 5D). Notably, the half-life of nivolumab in human serum is much longer (12-20 days) (27).

Nivolumab produces sustained cancer pain relief despite an initial increase in pain. To investigate the actions of nivolumab on bone cancer pain, we assessed mechanical, heat, and cold sensitivity before each i.v. injection on days $0,7,10$, and 14 (Figure 4A). Compared with human IgG4, nivolumab reduced hind-paw mechanical allodynia and knee hyperalgesia on days 7, 10, and 14 (Figure 4, $\mathrm{B}$ and $\mathrm{C}$ ). Meanwhile, nivolumab also decreased heat hyperalgesia and cold allodynia on days 7, 10, and 14 after LLC inoculation (Figure 4, D and E). Nivolumab-elicited pain relief was observed in both male and female mice (Supplemental Figure 3, B-E and G-J), and no sex differences were detected in bone cancer pain during the treatment (Supplemental Figure 4). We also tested the acute effects of nivolumab treatment on cancer pain. Nivolumab (10 mg/ kg., i.v.) evoked rapid increases in mechanical allodynia and thermal hyperalgesia at 3 hours after the injection on day 3 and day 7 after LLC inoculation (Supplemental Figure 6, A-E). Together with the data from $P d 1-\mathrm{KO}$ mice, our findings suggest that PD-1 blockade increases basal pain sensitivity.
Different tumor cell lines show distinct responses to anti-PD-1 treatment (28). We next tested to determine whether nivolumab could produce beneficial effects in bone metastasis induced by CMT-167 cells (29). Femoral inoculation of CMT-167 induced profound cancer pain (Supplemental Figure 7, A-C). Notably, nivolumab treatment also alleviated mechanical allodynia and cold allodynia at the early stages of tumor progression (Supplemental Figure 7, B and C). Furthermore, CMT-167-induced bone destruction was protected by the treatment (Supplemental Figure 7E).

Next, we assessed whether delayed nivolumab treatment would reduce cancer pain and bone destruction. We initiated nivolumab or human IgG treatment on day 5 after tumor inoculation, when bone pain and bone destruction were evident (Supplemental Figure 8, A-D). Nivolumab reduced mechanical allodynia on days 7 and 10, but not on day 14, after LLC inoculation (Supplemental Figure 8B). This treatment also improved bone destruction on days 8 and 11, but not on day 15 (Supplemental Figure 8, C and D). Nivolumab posttreatment only had a mild effect on bone fracture (Supplemental Figure 8E). Thus, posttreatment of nivolumab is also effective in reducing cancer pain and bone destruction, but with diminished efficacy in comparison with pretreatment. 
A

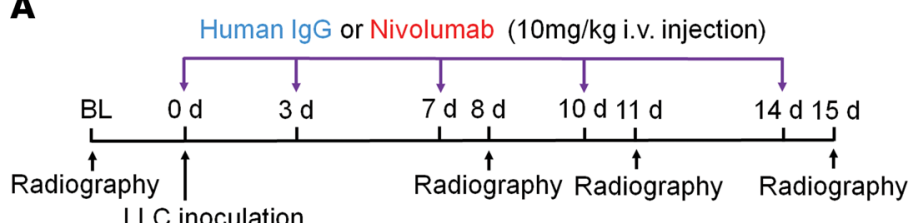

B
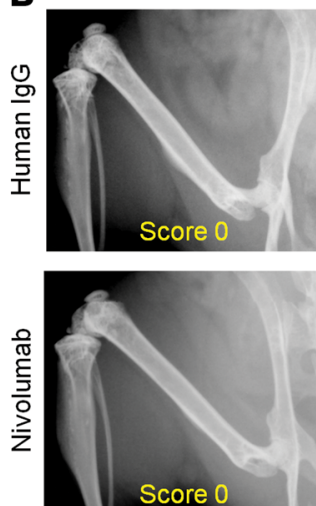

BL

E
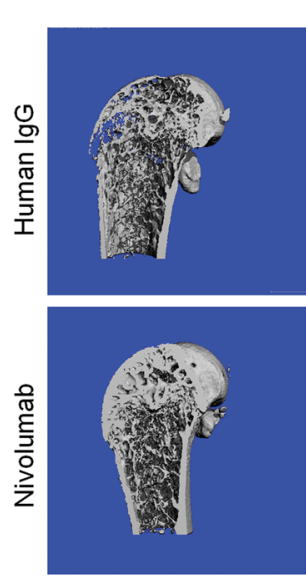


$8 d$


$11 \mathrm{~d}$
Time after LLC inoculation

$8 \mathrm{~d}$ after LLC inoculation

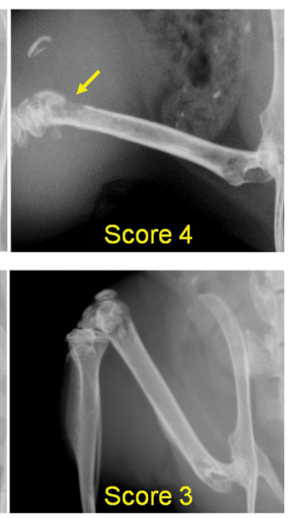

$15 d$
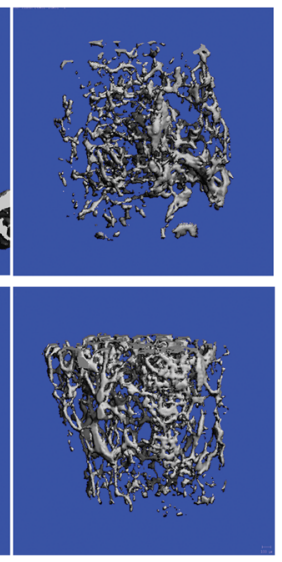

\section{C}

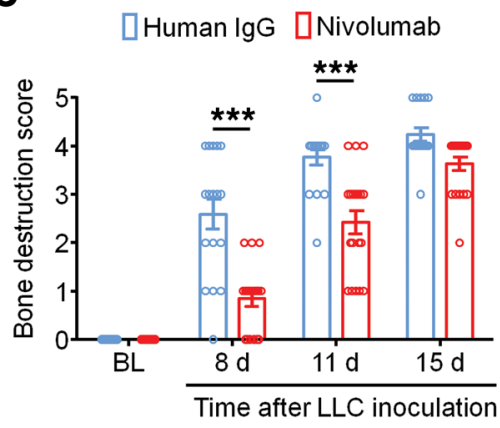

D

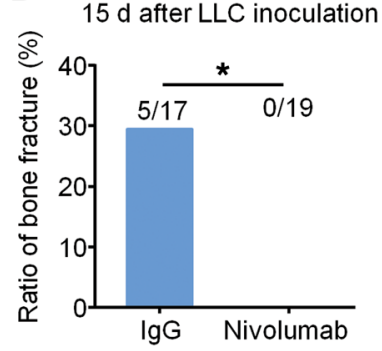

$\mathbf{F}$


Figure 3. Protection of cancer-induced bone destruction and fracture by i.v. nivolumab in tumor-bearing WT mice. (A) Experimental diagram for lgG or nivolumab injection (10 mg/kg, i.v.) and radiography testing. (B) Radiographs of tumor-bearing femora of IgG- or nivolumab-treated mice. Bone destruction score is shown in each photograph, and arrows indicate bone lesions with destruction scores over 3. (C) Quantification of bone destruction scores ( $n$ $=17$ or 19 mice). (D) Ratio of bone fracture of human IgG- and nivolumab-treated mice on day 15 ( $n=17$ or 19 mice). Note that bone fracture is protected by nivolumab. (E) Micro-CT images showing bone destruction in the distal part of tumor-bearing femora on day 8 after LLC inoculation. (F) Morphometric parameters from micro-CT showing higher BV/TV and Conn.D in distal part of ipsilateral femora in nivolumab group compared with human IgG group ( $n=$ 5-6 male mice) on day 8. Data are represented as mean \pm SEM. ${ }^{*} P<0.05$; ${ }^{* *} P<0.001$, repeated measures 2-way ANOVA with Bonferroni's post hoc test (C), Fisher's exact test (D), and 2-tailed Student's $t$ test (F).

PD-1 blockade has no effect on tumor burden after LLC inoculation. We assessed tumor burden by measuring the proportion of space occupied by tumor cells in BM 8 days after LLC inoculation. Surprisingly, nivolumab treatment did not reduce the tumor burden in femoral bone (Supplemental Figure 9, A and B). We also failed to see inhibition in tumor burden in $P d 1^{-/-}$mice on day 8 (Supplemental Figure 9, C and D). To further assess local tumor development, we established bone cancer by inoc- ulating luciferase-expressing LLC cells from the LL/2-Luc2 cell line. In vivo bioluminescence imaging showed that nivolumab did not change the total flux of tumor-bearing femurs when compared with IgG (Figure 4, F and G), arguing against an effect of nivolumab on local tumor growth in BM. Nivolumab produces anticancer effects via activation of $\mathrm{T}$ cells (15). We also evaluated the therapeutic effect of nivolumab in Rag1 $1^{-/-}$mice lacking mature $\mathrm{T}$ cells. We found that nivolumab treatment could still 
A Human IgG or Nivolumab (10mg/kg i.v. injection)

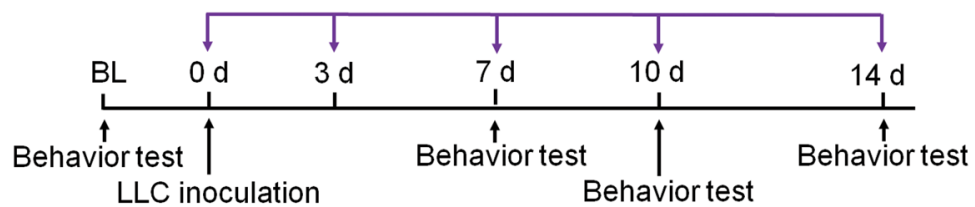

B

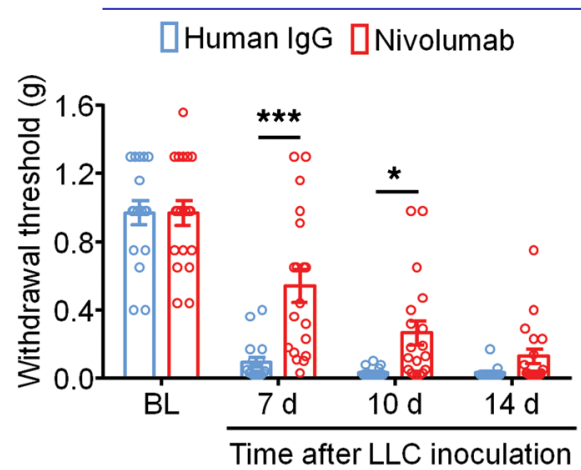

D

Heat sensitivity

DHuman IgG DNivolumab



$\mathbf{F}$
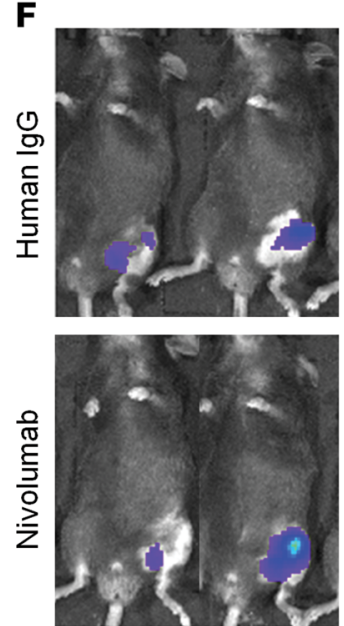

$8 d$
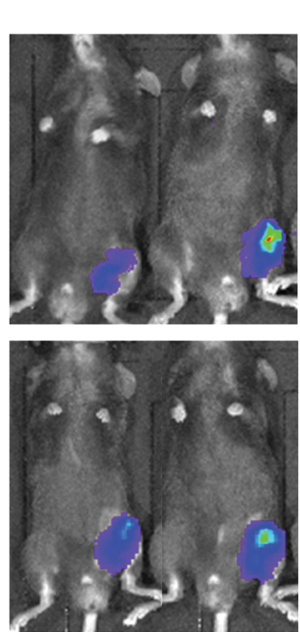

$11 \mathrm{~d}$

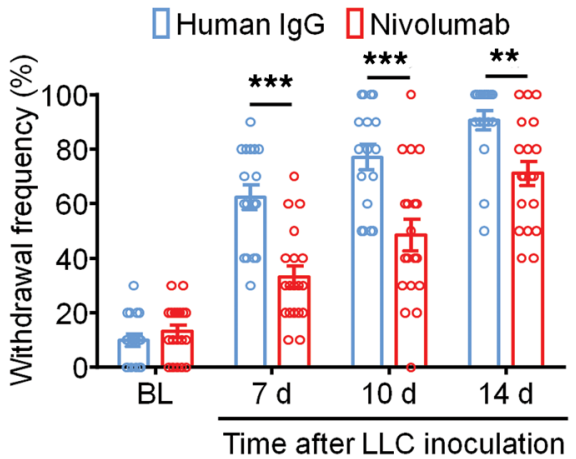

E

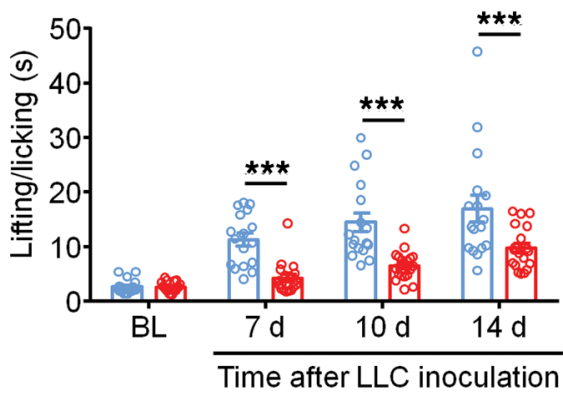

Cold allodynia

$\square$ Human IgG $\square$ Nivolumab

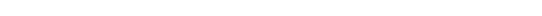

C Knee hyperalgesia

\Human IgG \Nivolumab

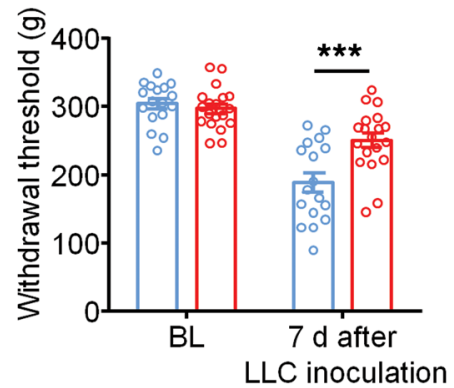

$\mathbf{G}$
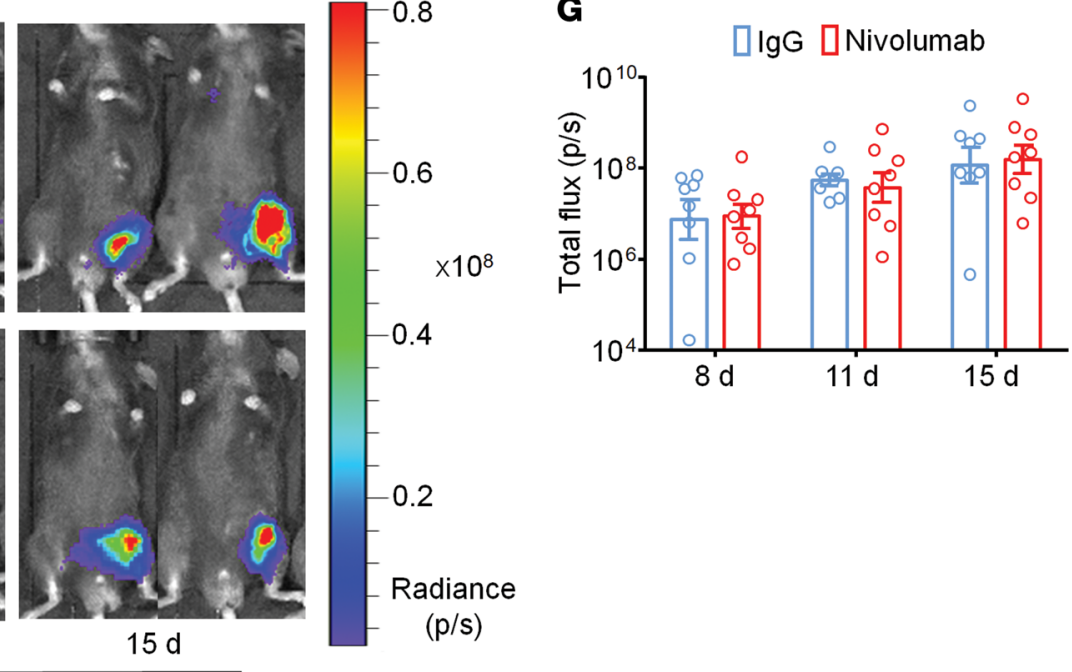

Time after LLC inoculation

Figure 4. Effects of nivolumab on bone cancer pain and tumor burden in tumor-bearing WT mice. (A) Experimental diagram for nivolumab or lgC treatment (10 mg/kg, i.v.) and behavioral tests. (B) von Frey test of withdrawal threshold (left) and frequency (right) ( $n=17$ or 19 mice). (C) PAM showing LLC-induced knee hyperalgesia in nivolumab- and human IgG-treated mice ( $n=17$ or 19 mice). ( $\mathbf{D}$ and $\mathbf{E})$ Hargreaves test (D) and acetone test (E) showing heat hyperalgesia (D) and cold allodynia (E) in human IgG- and nivolumab-treated mice ( $n=17$ or 19 mice). (F and $\mathbf{G}$ ) In vivo bioluminescence imaging showing no effects of nivolumab or human IgC ( $5 \times 10 \mathrm{mg} / \mathrm{kg}$, i.v.) on total flux of LL/2-Luc2 bearing femur on days 8,11 , and 15 after tumor inoculation ( $n=8$ mice). Images were acquired at 15 minutes after i.p. injection of $\mathrm{D}$-luciferin $(30 \mathrm{mg} / \mathrm{kg})$. Data are represented as mean $\pm \mathrm{SEM}$. ${ }^{*} P<0.05 ;{ }^{*} P<0.01 ;{ }^{* *} P<0.001$, repeated measures 2 -way ANOVA with Bonferroni's post hoc test. 

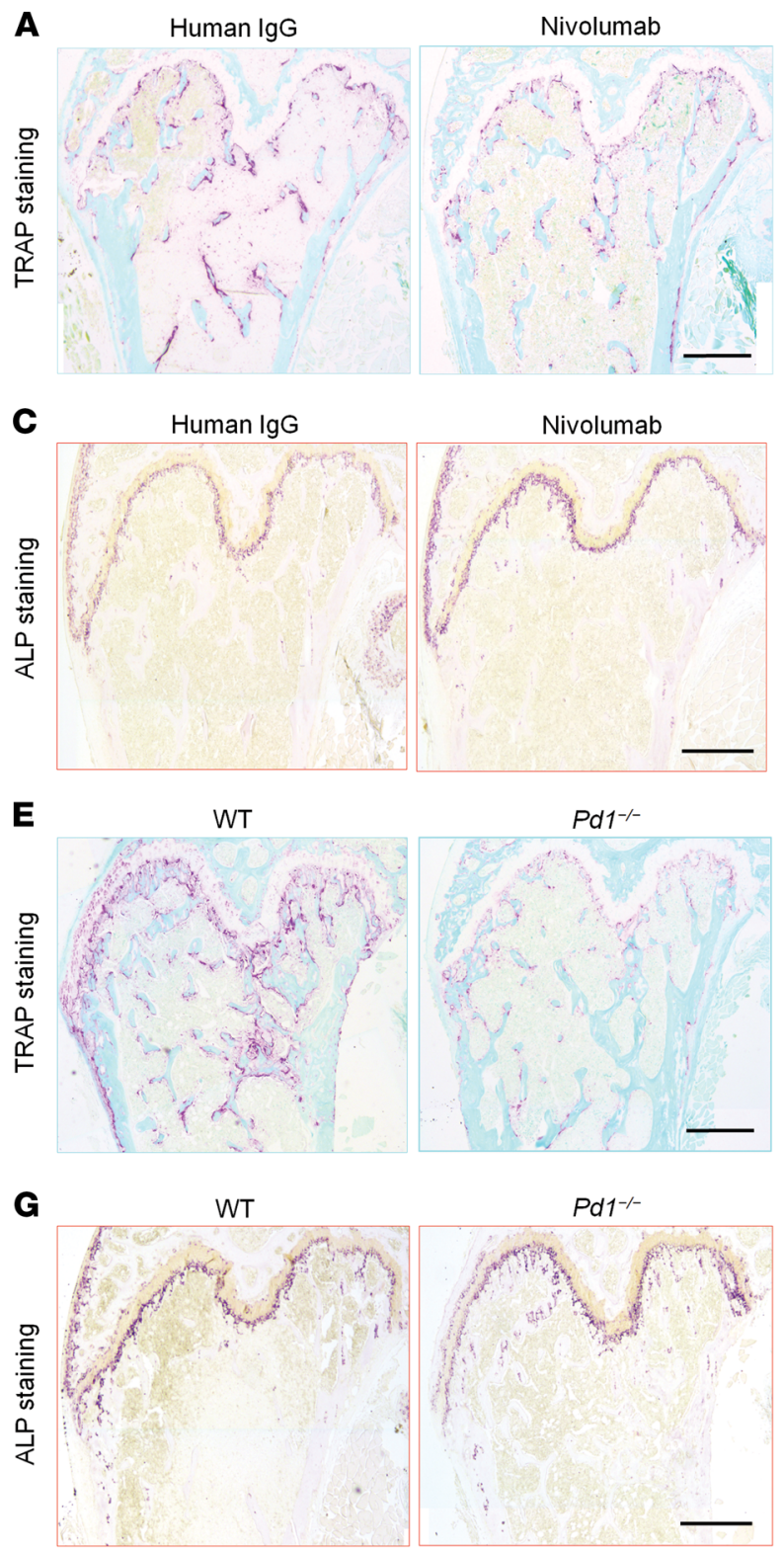


$\mathbf{F}$

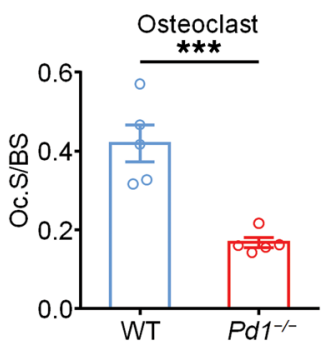

H



Figure 5. Effects of nivolumab or $P d 1$ deletion on TRAP' osteoclasts and ALP + osteoblasts in tumor-bearing femur. (A) Histological images of TRAP staining of osteoclasts in femurs from mice treated with human IgG or nivolumab (10 $\mathrm{mg} / \mathrm{kg}$, i.v.). Scale bar: $500 \mu \mathrm{m}$. (B) Quantification of TRAP staining in distal tumor-bearing femora (4-10 slices per femur, $n=5-6$ male mice). (C) Histological images for ALP staining of osteoblasts of femur bones from human IgG- or nivolumab-treated mice. Scale bar: $500 \mu \mathrm{m}$. (D) Quantification of ALP staining of osteoblasts in distal femora (4-10 sections per femur, $n=5$ male mice per group). (E and $\mathbf{F}$ ) Representative images $(\mathbf{E})$ and quantification of TRAP staining (F) in Pd1 ${ }^{-/}$mice and WT mice on postinoculation day 8 (4-10 slices per femur $n=5$ male mice). Scale bar: $500 \mu \mathrm{m}$. (G and $\mathbf{H})$ Representative images (G) and quantification of ALP staining $(\mathbf{H})$ showing osteoblasts in $P d 1^{-/}$mice and WT mice on postinoculation day 8 (4-10 slices per femur, $n=5$ male mice). Scale bar: $500 \mu \mathrm{m}$. (I and J) ELISA analysis showing the effects of nivolumab and IgG on serum levels of CTX-I (I) and PINP (J) on postinoculation day. $n=6-7$ male mice. Data are represented as mean \pm SEM. ${ }^{*} P<0.05 ;{ }^{* *} P<0.001$, 2-tailed Student's $t$ test (B, D, $\mathbf{F}$, and $\mathbf{H}$ ) and repeated measures 2-way ANOVA with Bonferroni's post hoc test (I and J). Oc.S/BS, osteoclast surface per trabecular bone surface; Ob.N/BS, osteoblast number per trabecular bone surface.

vironment. Tumor cells produce osteolytic bone lesions by producing osteoclast activators (30). To this end, we assessed whether nivolumab treatment would affect the formation of osteoclasts and osteoblasts in tumor-bearing BM 8 days after LLC inoculation. We observed that repeated i.v. nivolumab injections (Figure $4 \mathrm{~A}$ ) effectively reduced the formation of osteoclasts, as revealed by tartrateresistant acid phosphatase (TRAP) staining, in the distal part of tumor-bearing femora, where bone lesions first occurred (Figure 5, A and B). In contrast, nivolumab therapy did not alter the number of osteoblasts revealed by alkaline phosphatase (ALP) staining (31) of femoral sections (Figure 5, C and D). Consistently, tumor-bearing $\mathrm{Pd1}^{-/-}$mice also demonstrated decreased numbers of osteoclasts in the distal femurs (Figure

attenuate bone cancer pain in the von Frey test and bone destruction on days 7 and 8 after LLC inoculation (Supplemental Figure 10). This result indicates that bone protection by anti-PD-1 treatment does not require $\mathrm{T}$ cells.

Nivolumab inhibits TRAP $P^{+}$osteoclast differentiation in tumorbearing femur. Bone formation and destruction depend on homeostatic balance of osteoclasts and osteoblasts in the BM microen-
5, E and F). No difference was found in numbers of osteoblasts between tumor-bearing $P d 1^{-/-}$and WT mice (Figure 5, G and H). We also found no difference in the numbers of osteoclasts and osteoblasts between naive WT and $P d 1^{-/-}$mice (8-10 weeks, Supplemental Figure 11).

To further determine the activity of osteoclasts and osteoblasts after nivolumab or IgG treatment, we collected serum 
A

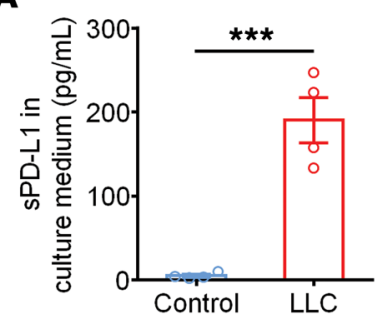

B $\square$ Human IgG $\square$ Nivolumab

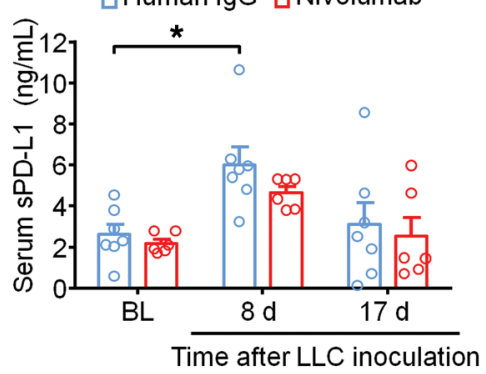

D



$8 \mathrm{~d}$ after LLC inoculation

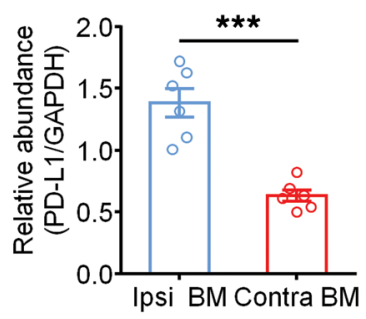

$\mathbf{F}$

PD-L1/DAPI



Contra BM

C



SSC-A

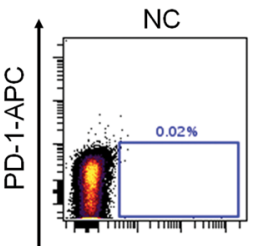

Gate2

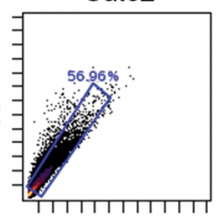

FSC-H



PD-L1-PE



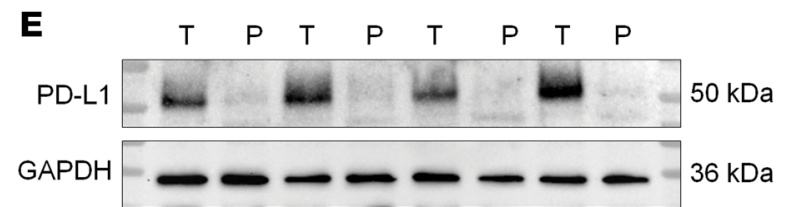

$17 \mathrm{~d}$ after LLC inoculation

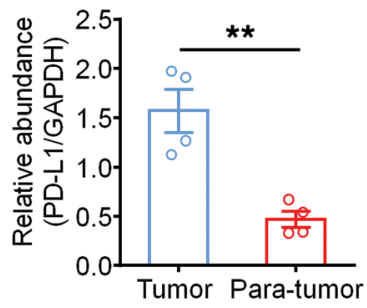

G

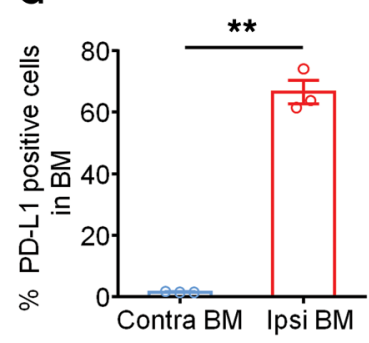


Figure 6. Bone cancer is associated with increased expression and secretion of PD-L1 in tumor-bearing mice. (A) SPD-L1 in culture medium of LLC or control (medium only without cells) revealed by ELISA. 1-1.5 × $10^{6}$ cells were included per well. $n=4$ cultures. (B) Increased serum SPD-L1 levels after tumor inoculation and the effects of human IgG and nivolumab. $n=6-7$ male mice. (C) Flow cytometry analysis showing serum increase in $\mathrm{PD}-\mathrm{L} 1^{+}$exosomes on day 8 after tumor inoculation. $n=4$ male mice. NC, negative control. (D) Western blot revealing PD-L1 expression in ipsilateral (I) and contralateral (C) BM collected 8 days after tumor implantation. Top: representative Western blot bands. Bottom: quantification of PD-L1 expression levels. $n=6$ male mice. (E) Western blot showing PD-L1 expression in tumor tissue of ipsilateral thigh $(T)$ or paratumor tissue $(P) 17$ days after LLC inoculation. Top: Western blot bands. Bottom: quantification of PD-L1 expression. $n=4$ male mice. (F) Immunostaining images showing PD-L1 expression in ipsilateral BM (Ipsi BM) and contralateral BM (Contra BM). Scale bar: $1000 \mu \mathrm{m}$. Low-magnification images on the left and right are enlarged in middle boxes. Scale bars: $50 \mu \mathrm{m}$. (G) Quantification of the percentage of $\mathrm{PD}-\mathrm{L} 1^{+}$cells in BM. $n=3$ male mice. Data are represented as mean \pm SEM. ${ }^{*} P<0.05$; ${ }^{* *} P<0.01$; ${ }^{* *} P<0.001$, 2-tailed Student's $t$ test $(\mathbf{A}, \mathbf{C}, \mathbf{D}, \mathbf{E}$, and $\mathbf{G})$ and repeated measures 2-way ANOVA with Bonferroni's post hoc test (B).

from tumor-bearing mice on day 8 and day 17 after LLC inoculation and measured serum PINP and CTX-I proteins, which are markers for bone formation and bone resorption, respectively (32). Nivolumab inhibited the serum CTX-I levels, which were increased on day 8 after tumor inoculation, but the serum PINP levels were not affected by nivolumab (Figure 5, I and J). Thus, nivolumab treatment could reduce destructive osteoclast activation in murine bone cancer.

Bone cancer is associated with increased PD-L1 and PD-1 expression. PD-L1 expression in the tumor microenvironment determines the response and efficacy of anti-PD-1 treatment (28). We assessed PD-L1 expression and secretion using different approaches. First, ELISA showed increased secretion of soluble PD-L1 (sPD-L1) (Figure 6A) in culture medium of LLC cells collected at 24 hours $\left(1-1.5 \times 10^{6}\right.$ cells per well). Second, ELISA showed increased serum sPD-L1 levels 8 days after LLC inoculation (Figure 6B). Third, we observed a marked increase in serum PD-L1 ${ }^{+}$exosomes by flow cytometry (Figure 6C). Cancer cells are known to release exosomes with surface expression of PD-L1 to regulate immunosuppression (33). Fourth, Western blotting showed increased expression of PD-L1 in ipsilateral cancer-bearing BM compared with contralateral cancer-absent BM (Figure $6 \mathrm{D})$. On day 17 , tumor growth outside the bone cavity was evident, but tumor tissue has a higher expression of PD-L1 compared with paratumor tissue (Figure 6E). Finally, immunohistochemistry revealed increased expression of PD-L1 in cancer-bearing BM (Figure 6, F and G). PD-L1 mainly expressed on the cell surface of tumor cells, with irregular and larger nuclei compared with normal BM cells (Figure 6F).

Next, we investigated PD-1 expression using immunohistochemistry and flow cytometry (Figure 7). Immunostaining showed basal PD-1 expression in normal BM cells and also revealed increased PD-1 expression in tumor-bearing BM (Figure 7A). Double staining demonstrated that both PD-L1 and PD-1 had higher expression in cancer-bearing BM (Figure $7 \mathrm{~B})$. As monocytes and macrophages are preosteoclasts in BM (34), we further used flow cytometry to characterize PD-1 expression in BM. PD-1 expression levels increased in both monocytes and macrophages in ipsilateral BM with tumor compared with contralateral cancer-free BM (Figure 7, $C$ and D). These findings indicate a high expression of PD-1 in preosteoclasts in tumor-bearing femur.

$P D-L 1$ promotes RANKL-induced osteoclastogenesis via JNK activation. We further determined the role of PD-L1 and PD-1 axis in osteoclast differentiation in vitro. We used RAW 264.7 cells of murine macrophages and induced osteoclast formation with a low dose of RANKL ( $20 \mathrm{ng} / \mathrm{mL}$, for 6 days). Incubation of RAW 264.7 cells with $1 \mu \mathrm{g} / \mathrm{mL}$ nivolumab $(7 \mathrm{nM}$ ) did not alter the number of osteoclasts as defined by $\mathrm{TRAP}^{+}$multinucleated cells (Figure 8, A and B). Notably, PD-L1 (100 ng/mL) and IgG (1 $\mu \mathrm{g} / \mathrm{mL}$ ) cotreatment could promote osteoclastogenesis, which could be blocked by PD-L1 (100 ng/mL) and nivolumab $(1 \mu \mathrm{g} /$ $\mathrm{mL}$ ) cotreatment (Figure 8, A and B). Therefore, PD-L1 induced osteoclastogenesis via PD-1 in the presence of RANKL. To validate a critical role of PD-L1 in osteoclastogenesis, we next harvested BM cells and differentiated them into osteoclasts with 20 $\mathrm{ng} / \mathrm{mL}$ MCSF and $20 \mathrm{ng} / \mathrm{mL}$ RANKL for 7 days. Consistently, PD-L1 $(100 \mathrm{ng} / \mathrm{mL})$ increased osteoclast differentiation, but this effect was blocked by nivolumab $(1 \mu \mathrm{g} / \mathrm{mL}$, Figure $8, \mathrm{C}$ and D). We also harvested BM cells from WT and $P d 1^{-/-}$mice for the induction of osteoclasts and observed that PD-L1 failed to affect osteoclast differentiation in Pd1-null BM cells (Figure 8, E and F). These data further confirm that PD-L1 enhances osteoclastogenesis via PD-1.

To investigate a time-dependent expression of PD-L1 and PD-1, we induced osteoclastogenesis from RAW 264.7 cells or isolated BM cells and collected cells at 1 day, 3 days, 5 days, or 7 days after RANKL treatment. Double-immunostaining results showed dynamic expression patterns of PD-L1 and PD-1. We observed persistent PD-L1 expression at every stage of cell growth, but also found decreased PD-1 expression during osteoclastogenesis (Supplemental Figure 12, A and B). In particular, during the induction of osteoclasts from primary BM cells, PD-1 expression was almost lost (Supplemental Figure 12B). These results strongly indicate that anti-PD-1 treatment specifically targets the differentiation stage from preosteoclasts to osteoclasts, without altering the function of mature osteoclasts.

MAPKs, including of ERK, p38, and JNK, are involved in RANK/RANKL signaling, osteoclast differentiation, and pathogenesis of pain $(35,36)$. In the presence of RANKL, PD-L1 $(100$ $\mathrm{ng} / \mathrm{mL}$ ) increased the phosphorylation of JNK (p-JNK), without affecting p-ERK and p-p38 levels in BM-derived macrophages (BMDM) (Figure 8, $\mathrm{G}$ and $\mathrm{H}$ ); also, the p-JNK increase was blocked by nivolumab $(1 \mu \mathrm{g} / \mathrm{mL}$, Figure $8 \mathrm{I}$ and Supplemental Figure 15$)$. Moreover, the JNK pathway inhibitor SP600125 $(10 \mu \mathrm{M})$ suppressed osteoclastogenesis induced by PD-L1 $(100 \mathrm{ng} / \mathrm{mL})$ (Figure $8, \mathrm{~J}$ and $\mathrm{K}$ ). Thus, $\mathrm{PD}-\mathrm{L} 1$ may promote osteoclastogenesis via the JNK/MAPK signaling pathway.

CCL2 and CCR 2 regulate osteoclast differentiation and cancer pain. To further investigate the mechanism by which PD-L1 causes osteoclast activation in bone cancer pain, we employed a cytokine array to examine the expression of cytokines and chemokines during osteoclast differentiation (37). We incubated the blot coated with cytokine antibodies with culture medium from BMDM treated with MCSF (20 ng/mL, 24 hours) and RANKL (40 ng/ 
A

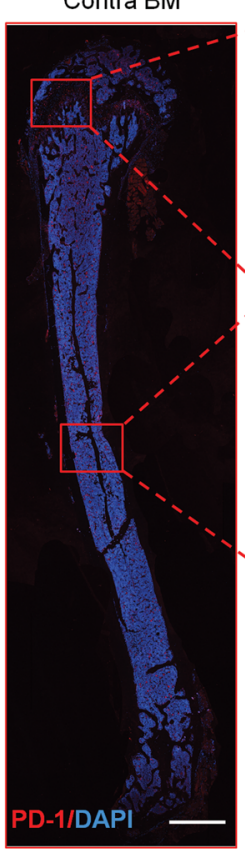



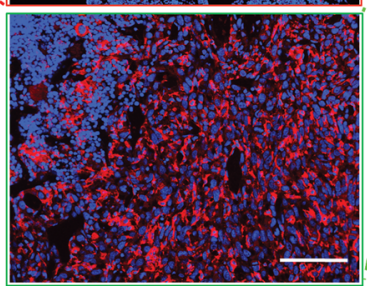

Ipsi BM

C



Gate 2

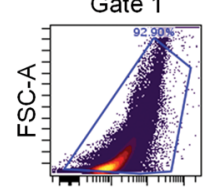

SSC-A

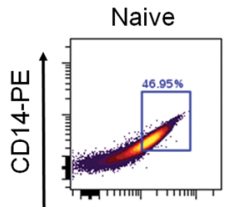

FSC-H


PD1-680

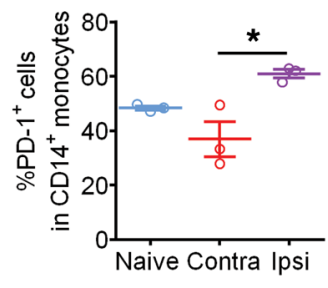

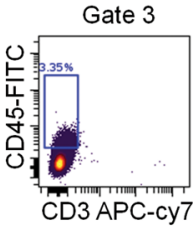

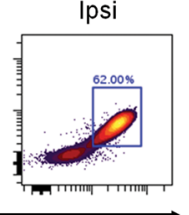

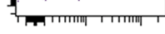

B
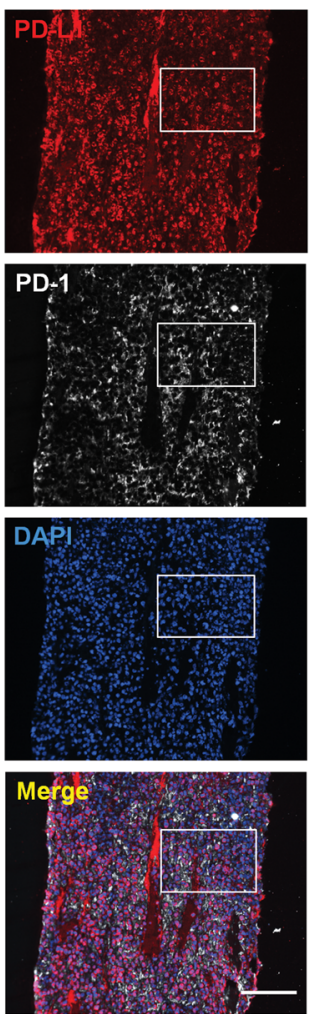
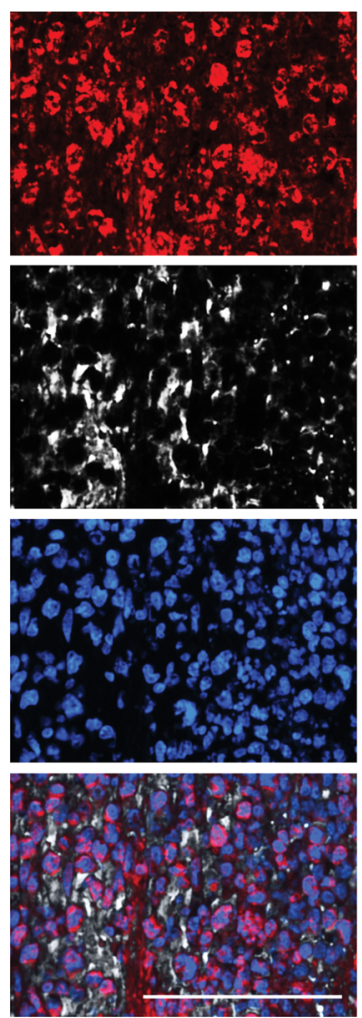

D


Ipsi



PD1-680

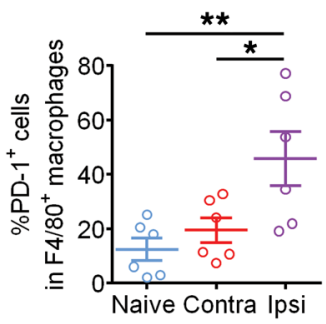

Figure 7. Bone cancer is associated with increased PD-1 expression in tumor-bearing femora. (A) Immunostaining images showing PD-1 expression in ipsilateral BM and contralateral BM on 8 days after inoculation. Scale bar: $1000 \mu \mathrm{m}$. Note the sample of contralateral BM here is the same as the contralateral BM shown in Figure 6F. Low-magnification images on the left and right are enlarged in middle boxes showing PD-1 staining in normal BM environment and in tumor microenvironment. Scale bars: $200 \mu \mathrm{m}$ (contralateral BM); $100 \mu \mathrm{m}$ (ipsilateral BM). (B) Double staining of PD-L1 (red) and PD-1 (white) in ipsilateral BM on day 8. The boxes in the left panels are enlarged in the right panels. Scale bars: $100 \mu \mathrm{m}$. (C and D) Increased expression of PD-1 in monocytes (C) and microphages (D) in ipsilateral BM inoculated with tumor compared with contralateral BM and BM of naive mice. Monocytes were labeled as CD3-, $\mathrm{CD}_{45}{ }^{+}$, and $\mathrm{CD} 14^{+}$cells, and macrophages were identified as F4/80+ cells. Top: representative images of flow cytometry. Bottom: quantification of percentages of PD-1+ cells in monocyte and macrophage populations. $n=3-6$ mice. Data are represented as mean \pm SEM. ${ }^{*} P<0.05$; ${ }^{*} P<0.01,1$-way ANOVA with Bonferroni's post hoc test. 
$\mathrm{mL}, 24$ hours). Compared with vehicle, this treatment induced a marked increase in IP-10, sICAM-1, IL-1ra, MIP-1 $\beta$, and CCL2 (Supplemental Figure 13, B and C). Since CCL2 plays a crucial role in inflammatory and neuropathic pain via the CCR2 receptor $(38,39)$, we further analyzed CCL2/CCR2 signaling in BM and osteoclasts. ELISA analysis verified CCL2 upregulation in culture medium during preosteoclast to osteoclast transition (Figure 9A). Since JNK regulates CCL2 release in astrocytes (37), we next tested to determine whether PD-L1 would regulate CCL2 release from BMDM via JNK signaling. PD-L1 (100 ng/mL, 1 hour) elevated CCL2 in culture medium, but this increase was blocked by SP600125 (20 $\mu \mathrm{M}$, Figure 9B). CCL2 secretion by PD-L1 was also blocked by nivolumab ( $1 \mu \mathrm{g} / \mathrm{mL}$, Figure $9 \mathrm{C})$. We observed a marked increase in CCL2 levels in ipsilateral tumor-bearing BM compared with contralateral tumor-free $\mathrm{BM}$ in mice 8 days after LLC inoculation (Figure 9D). Taken together, our results suggest that PD-L1 could increase CCL2 release from BMDM and that this process requires $\mathrm{PD}-1$ and $\mathrm{JNK}$.

Next, we investigated whether CCL2 signaling regulates osteoclastogenesis and cancer pain. CCL2 $(50 \mathrm{ng} / \mathrm{mL})$ promoted osteoclastogenesis in BM cultures in the presence of low-dose RANKL (20 ng/mL), but this effect was abolished by CCR2 antagonist RS $504393(1 \mu \mathrm{g} / \mathrm{mL}$, Figure 9E). We used a sensitive $\mathrm{Ca}^{2+}$ indicator, GCaMP6, to assess calcium signaling in dissociated DRG neurons from advillin-GCaMP6 mice. We observed dose-dependent $\mathrm{Ca}^{2+}$ responses in DRG neurons following CCL2 stimulation $(50,100$, and $450 \mathrm{ng} / \mathrm{mL}$, Figure 9F). CCL2 induced a weak $\mathrm{Ca}^{2+}$ signal at $50 \mathrm{ng} / \mathrm{mL}(\sim 6 \mathrm{nM})$, but strong signals at higher doses in DRG neurons that also responded to capsaicin, suggesting that CCL2 selectively activates C-fiber nociceptive neurons (Figure 9F). In situ hybridization with RNAscope revealed Cor 2 mRNA expression in DRG neurons, with an upregulation in ipsilateral DRGs compared with contralateral DRGs in bone cancer pain (Figure 9, G and H). Since tumor-bearing bone is innervated by pain-sensing nerve fibers (13), CCL2 released from preosteoclasts and osteoclasts could directly act on CCR2 ${ }^{+}$ nociceptive fibers to elicit bone cancer (Figure 10). To test the contribution of CCL2/CCR2 signaling to cancer pain, we treated tumor-inoculated mice with RS 504393 (10 and $30 \mathrm{mg} / \mathrm{kg}$ ). We observed a dose-dependent inhibition of mechanical allodynia by RS $504393(30 \mathrm{mg} / \mathrm{kg})$ at 1, 3, or 6 hours after single i.p. injection (Figure 9I).

PD-1 blockade does not affect osteoclast formation and bone microstructure in naive mice. To address the issue that immune activation by immunotherapy may result in loss of bone mass, we evaluated the impact of nivolumab or human IgG treatment on osteoclastogenesis and bone microstructure in naive mice using the same medication (Figure 4A). We collected femurs for microCT scanning and immunostaining on day 17. Notably, nivolumab did not modify the formation of osteoclasts and osteoblasts in naive mice, as revealed by TRAP and ALP staining (Supplemental Figure 14, A and B). Also, the morphometric parameters from micro-CT analysis of nivolumab- and IgG-treated mice showed no difference in BV/TV, Conn.D, trabecular thickness (Tb.Th), trabecular separation (Tb.Sp), and trabecular number (Tb.N) (Supplemental Figure 14, C and D). Therefore, our nivolumab treatment does not affect the normal structure of bone.

\section{Discussion}

In this study, we have demonstrated how PD-1 regulates bone cancer pain following femur inoculation of LLC. We found paradoxical regulations of cancer pain by PD-1 via distinct cellular mechanisms. In agreement with our previous study (19), loss of PD-1 led to increased pain sensitivity, as neuronal PD-1 negatively regulates nociceptor excitability (19). Therefore, PD-1 blockade with i.v. nivolumab caused an initial enhancement of bone cancer pain at 3 hours after each injection. Importantly, persistent bone cancer pain was protected in $P d 1^{-/-}$mice 7 days after treatment. The i.v. nivolumab also produced sustained relief of bone cancer pain in WT mice. This long-term pain relief by PD-1 inhibition is operated by a mechanism through inhibition of PD-L1/CCL2-mediated osteoclastogenesis and protection of bone destruction. It is striking to note that bone fracture during tumor progression was abolished after $P d 1$ deletion or nivolumab treatment.

Our finding shows that cancer-produced PD-L1 promoted the osteoclastogenesis via PD-1 in the tumor BM microenvironment (Figure 10). We observed increased PD-L1 expression in tumor-bearing BM 8 days after LLC inoculation, when osteoclast differentiation and bone lesion became evident. Upregulation of PD-L1 in BM tumor cells was associated with increased PD-L1 secretion in serum during tumor progression. Interestingly, we also found PD-L1 upregulation in secreted exosomes, which are involved in immunosuppression (33). Additionally, PD-1 was highly expressed in preosteoclasts, including monocytes and macrophages, and PD-1 expression was lost during osteoclast formation in vitro. We also provided several lines of evidence to support a direct involvement of the PD-L1/PD-1 axis in regulating osteoclast formation. First, $\mathrm{x}$-ray analysis revealed time-dependent bone destruction. This osteoclast-mediated bone destruction was protected by the anti-PD-1 treatment or Pd1 deletion. Second, TRAP staining in tumor-bearing BM and serum CTX-I analysis in serum demonstrated functionality of PD-1 blockade in suppressing osteoclast formation and osteoclast overactivation. Finally, in vitro studies also showed that exogenous application of PD-L1 could enhance low-dose RANKL-induced osteoclast differentiation in a nivolumab-sensitive manner. It is conceivable that PD-L1/PD-1-mediated osteoclastogenesis could drive bone destruction and the pathogenesis of bone cancer pain.

It is also interesting to note that CCL2 was upregulated by PD-L1 activation of JNK during osteoclast differentiation and contributed to osteoclastogenesis and bone cancer pain. CCL2, also called monocyte chemoattractant protein-1 (MCP-1), regulates murine and human osteoclast formation (40, 41). The CCL2/ CCR2 axis also plays a critical role in generating inflammatory and neuropathic pain $(38,42)$. We further demonstrated an involvement of this axis in cancer-induced bone pain. Through cytokine screening and ELISA analysis, we found increased CCL2 secretion by both preosteoclasts and mature osteoclasts in the presence of RANKL. Notably, our calcium imaging revealed CCL2 as a potent activator of nociceptors that respond to capsaicin. Additionally, CCR2 expression was increased in DRG neurons in bone cancer and anti-CCR2 treatment effectively attenuated mechanical allodynia in mice with bone cancer. These findings support crucial roles of the CCL2/CCR2 pathway in PD-L1-induced osteoclast activation and bone cancer pain (Figure 10). 
A

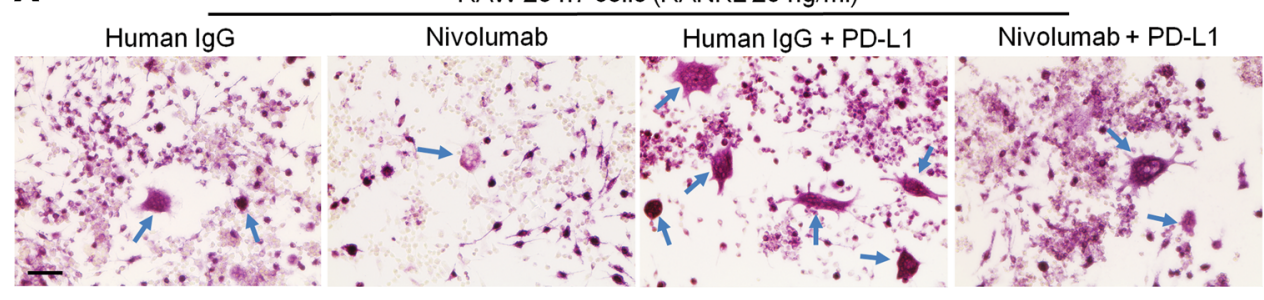

C

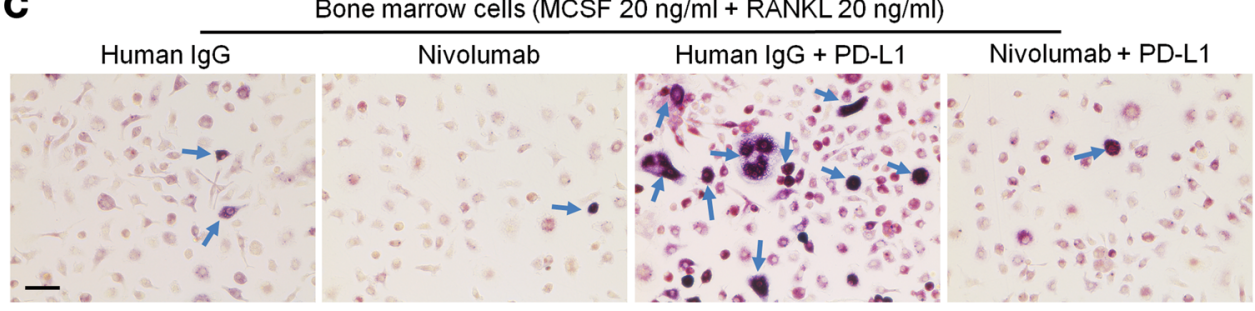

E

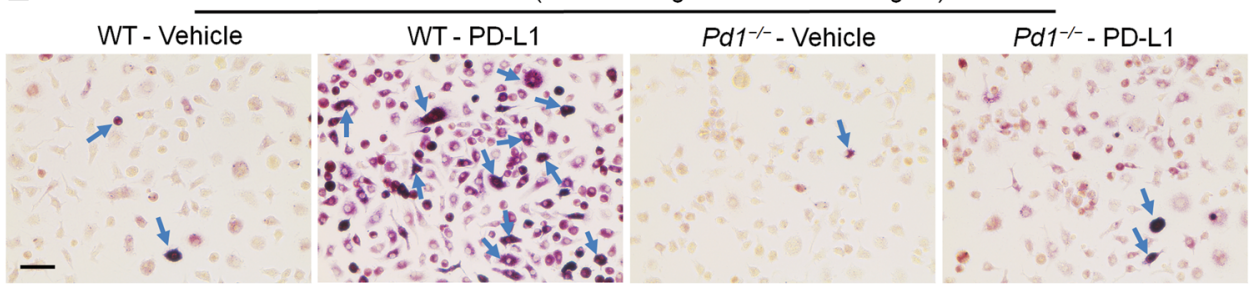

G

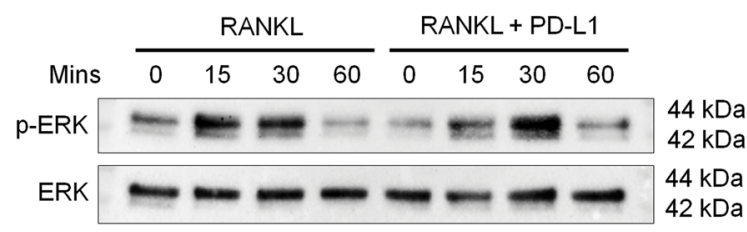

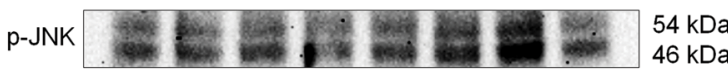

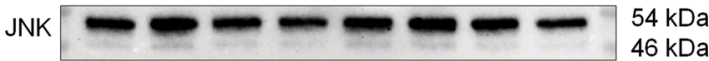

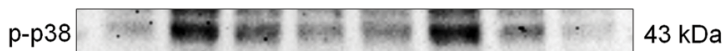

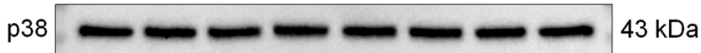

GAPDH

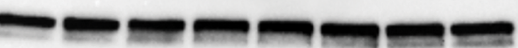

$36 \mathrm{kDa}$
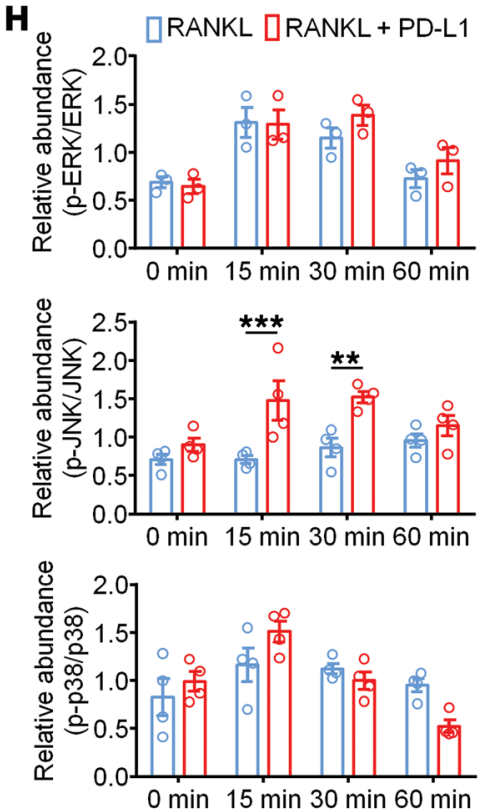
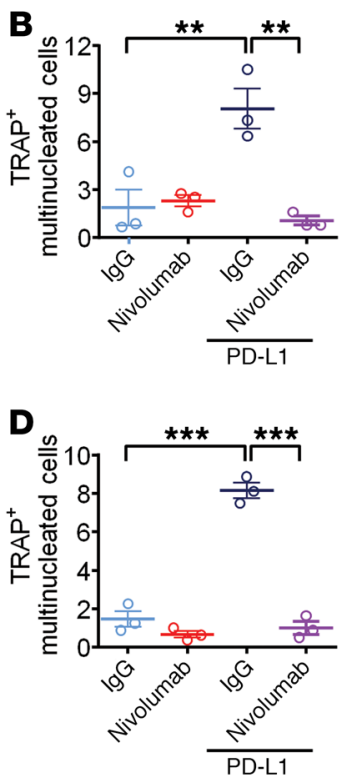

$\mathbf{F}$

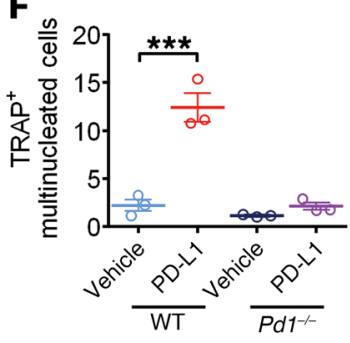

I

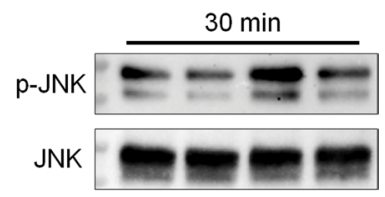

$\begin{array}{rllll}\text { RANKL } & + & + & + & + \\ \text { IgG } & \pm & - & \pm & \frac{1}{2} \\ \text { Nivolumab } & \pm & \pm & + & + \\ \text { PD-L1 } & - & - & + & +\end{array}$



$\mathbf{K}$

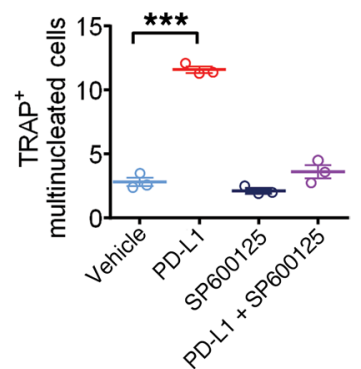

Bone marrow cells (MCSF $20 \mathrm{ng} / \mathrm{ml}+$ RANKL $20 \mathrm{ng} / \mathrm{ml}$ ) SP600125

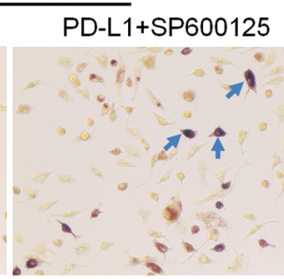


Figure 8. RANKL-induced osteoclastogenesis is promoted by PD-L1 via JNK. (A) Representative images of RAW 264.7 cells stained for TRAP. Cells were stimulated with low-dose RANKL $(20 \mathrm{ng} / \mathrm{mL})$ and coincubated with human IgG $(1 \mu \mathrm{g} / \mathrm{mL})$, PD-L1 (100 $\mathrm{ng} / \mathrm{mL})$, or nivolumab $(1 \mu \mathrm{g} / \mathrm{mL})$ for 6 days. Arrows indicate TRAP ${ }^{+}$multinucleated osteoclasts. Scale bar: $50 \mu \mathrm{m}$. (B) Quantification for A. $n=3$ cultures. (C) Representative images of TRAP staining for BM cultures treated with human IgG $(1 \mu \mathrm{g} / \mathrm{mL})$, PD-L1 (100 ng/ $\mathrm{mL})$, or nivolumab $(1 \mu \mathrm{g} / \mathrm{mL})$ for 6 days. MCSF, $20 \mathrm{ng} / \mathrm{mL}$; RANKL, $20 \mathrm{ng} /$ $\mathrm{mL}$. Arrows indicate TRAP ${ }^{+}$multinucleated osteoclasts. Scale bar: $50 \mu \mathrm{m}$. (D) Quantification for C. $n=3$ cultures. (E and $\mathbf{F}$ ) Representative images (E) and quantification (F) of TRAP staining for primary BM cultures from WT mice or Pd1/- mice treated with vehicle or PD-L1 $(100 \mathrm{ng} / \mathrm{mL}) . n=3$ cultures. Scale bar: $50 \mu \mathrm{m}$. (G and $\mathbf{H})$ Western blot showing p-ERK, p-JNK, and p-p38 in BMDM treated with RANKL $(20 \mathrm{ng} / \mathrm{mL}$ ) or RANKL (20 ng/ $\mathrm{mL}$ ) together with PD-L1 $(100 \mathrm{ng} / \mathrm{mL})$ at different time points. (G) Representative Western blot bands. (H) Quantification for $\mathbf{G}$. $n=3-4$ cultures from 3 or 4 male mice. (I) Western blot showing the effect of nivolumab on PD-L1-enhanced phosphorylation of JNK. $n=3$ cultures from 3 male mice. (J and $\mathbf{K}$ ) Representative images (J) and quantification (K) of TRAP staining of primary BM cultures treated with PD-L1 $(100 \mathrm{ng} / \mathrm{mL})$ or SP600125 $(10 \mu \mathrm{M})$. MCSF, $20 \mathrm{ng} / \mathrm{mL}$; RANKL, $20 \mathrm{ng} / \mathrm{mL}$. Scale bar: $50 \mu \mathrm{m}$. Data are represented as mean $\pm \mathrm{SEM}$. ${ }^{* *} P<0.01 ;{ }^{* *} P<0.001$, 1-way ANOVA with Bonferroni's post hoc test (B, D, F, I, and $\mathbf{K}$ ) and 2-way ANOVA with Bonferroni's post hoc test $(\mathbf{H})$.

Surprisingly, nivolumab treatment did not reduce tumor burden in BM during tumor progression. In Rag1 $1^{-/-}$mice lacking mature $\mathrm{T}$ cells, nivolumab was still able to attenuate bone destruction and cancer pain. This result further suggests that nivolumab protection of bone destruction relies on its direct suppression on osteoclast formation. Lack of tumor inhibition by our anti-PD-1 treatment may result from lower sensitivity of LLC to nivolumab, as different cell lines respond differently to immunotherapies in murine models (27).

This study has strong clinical implications, as nivolumab is approved by the FDA for treating advanced lung cancer as well as melanoma and head and neck cancers (16-18). Patients with bone metastasis have high risk of bone cancer pain. Thus, demonstrating the effect of nivolumab in preclinical models will guide future clinical trials in preventing and treating bone fractures and bone cancer pain. Because the half-life of nivolumab is much longer in humans than mice ( 2 weeks vs. 3 days), nivolumab could be more effective in protecting bone destruction and alleviating bone cancer pain in humans. Our finding also suggests nivolumab treatment does not disrupt normal bone structure in cancer-free animals. Thus, abnormal PD-L1 expression in BM microenvironment could be a prerequisite for this immunotherapy to be effective. Furthermore, our results indicate that posttreatment with nivolumab, starting 5 days after tumor inoculation, is still efficacious in decreasing bone destruction and cancer pain, despite better effects of prophylactic treatment. In addition to lung cancers, bone metastasis also occurs in latestage breast, thyroid, and bladder cancers as well as other cancers, and osteolytic bone lesions are very common in these cancers, leading to pathological fractures and severe bone cancer pain (4). It is noteworthy that improving life quality in terminal cancer patients is as important as extending survival. Thus, our discovery of protecting bone fractures and alleviating bone cancer pain with anti-PD-1 immunotherapy is innovative and highly feasible. Actually, several clinical studies have shown that anti-
PD-1 treatment might reduce cancer pain and improve quality of life in patients with advanced non-small-cell lung cancer and advanced head and neck cancer $(43,44)$. Case reports also indicate that nivolumab treatment can alleviate cancer pain in head and neck cancer patients with spinal bone metastasis and in renal cell carcinoma patients with multiple bone metastasis $(45,46)$. In particular, Ansari et al. showed a marked improvement in pain score, with a reduction from $8 / 10$ to $3 / 10$ (46). However, pain is not well assessed in these studies by pain specialists. It is unclear whether pain relief is a result of antitumor or bone-modification effect by anti-PD-1 treatment. Future studies are needed to specifically address this issue. Future studies are also warranted to test additional immunotherapy agents, such as PD-L1 and anti-CTLA4 antibodies, in different bone cancer conditions.

\section{Methods}

Reagents. The humanized anti-PD-1 antibody nivolumab (Opdivo) was purchased from Bristol-Myers Squibb. Mouse PD-L1 (catalog ab180058) and human IgG4 (catalog ab90286) were purchased from Abcam. Mouse RANKL protein (catalog 462-TEK), mouse M-CSF (catalog 416-ML), mouse CCL2/JE/MCP-1 protein (catalog 479-JE), and RS504393 (catalog 2517/10) were purchased from R\&D Systems. SP600125 (catalog 420119) was from MilliporeSigma.

Animals. Adult mice (males and females, 8-10 weeks) were used for behavioral and biochemical studies. C57BL/6 mice, PD-1 (Pdcd1) KO mice (catalog 021157), and Rag 1(Rag1) KO mice (catalog 002216) with a C57BL/6 background were purchased from the Jackson Laboratory and maintained at the Duke University animal facility. Advillin-GCaMP6 mice were generated by crossing GCaMP6f mice with Advillin-Cre mice (a gift from Fan Wang's lab, Duke University). From 2 to 5 mice were housed in each cage under a 12-hour light/12hour dark cycle with ad libitum access to food and water. Animals were randomly assigned to different experimental groups. Sample sizes were estimated based on our previous studies for similar types of behavioral and biochemical analyses $(19,25,47)$. Both males and females were used in a sex- and age-matched manner, unless otherwise specified in figure legends. The numbers of mice used in different experiments are summarized in Supplemental Table 1.

Cell culture. Murine LLC line LL/2 (LLC1) (CRL-1642), luciferaseexpressing cell line LL/2-Luc2 (CRL-1642-LUC2), and murine monocyte/macrophage cell line RAW 264.7 (TIB-71) were obtained from ATCC. Mouse lung carcinoma cell line CMT 167 (catalog 10032302) was obtained from the European Collection of Authenticated Cell Cultures (ECACC). Cells were cultured in high glucose $(4.5 \mathrm{~g} / \mathrm{L})$ DMEM (Gibco, Thermo Fisher Scientific). Culture media were supplemented with 10\% FBS (Gibco, Thermo Fisher Scientific) and 1\% antibioticantimycotic solution (MilliporeSigma) and placed in $5 \% \mathrm{CO}_{2} / 95 \%$ air at $37^{\circ} \mathrm{C}$. Blasticidin $(2 \mu \mathrm{g} / \mathrm{mL}$, Gibco, Thermo Fisher Scientific) was added into LL/2-Luc2 culture medium and was removed 3 days before the inoculation into mice. Cells were collected for experiments following enzymatic digestion with $0.05 \%$ trypsin.

Bone cancer pain model. Murine lung carcinoma cell line LLC1, LL/2-Luc2, or CMT 167 was digested with 0.05\% trypsin and made into a suspension of $5 \times 10^{7} / \mathrm{mL}$ cells in PBS. The inoculation was performed as previously described (48). Mice were anesthetized with $3 \%$ isoflurane, and a 0.5 to $1 \mathrm{~cm}$ superficial incision was made near the knee 

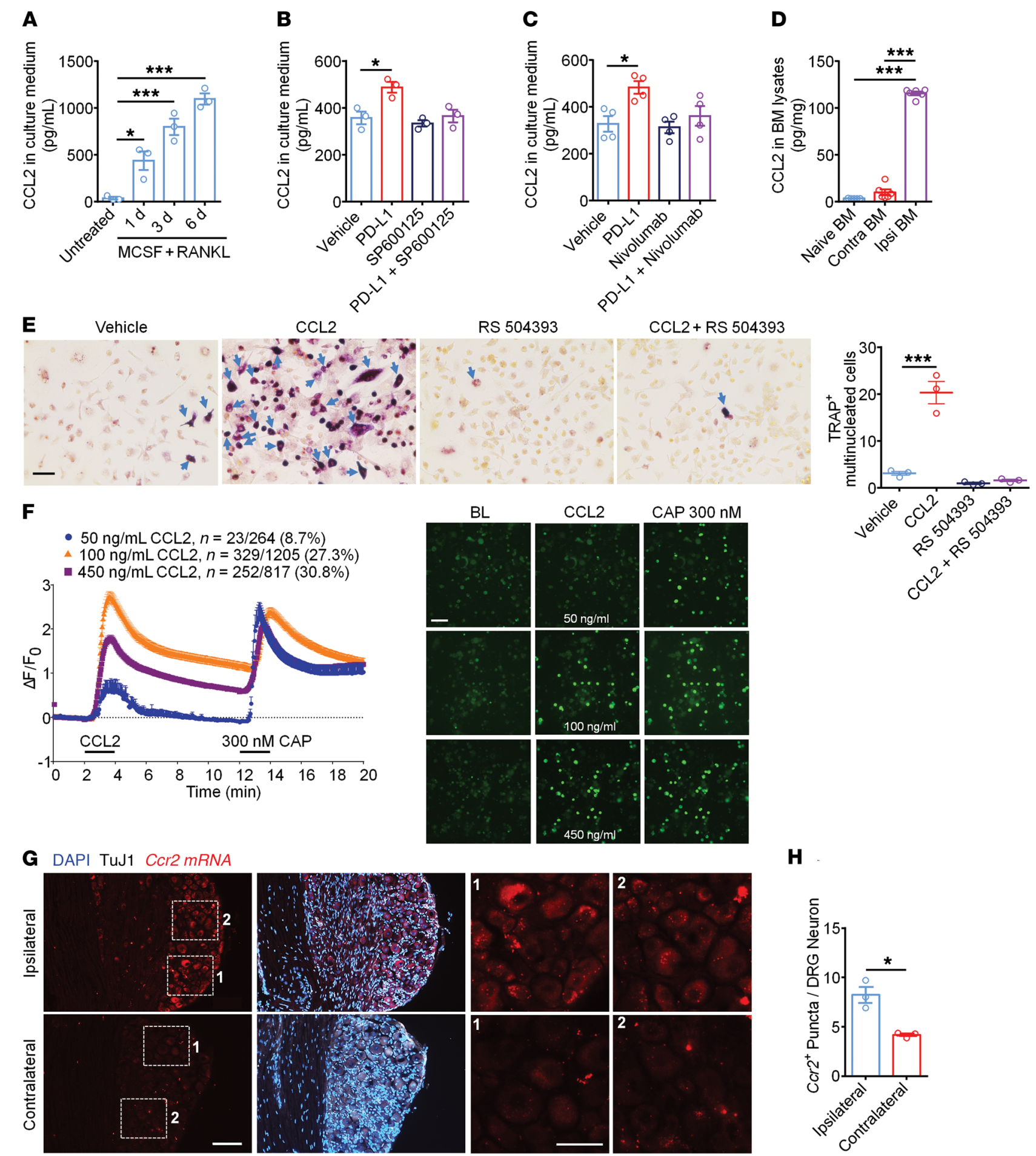

QVehicle RRS $50439310 \mathrm{mg} / \mathrm{kg}$ DRS $50439330 \mathrm{mg} / \mathrm{kg}$
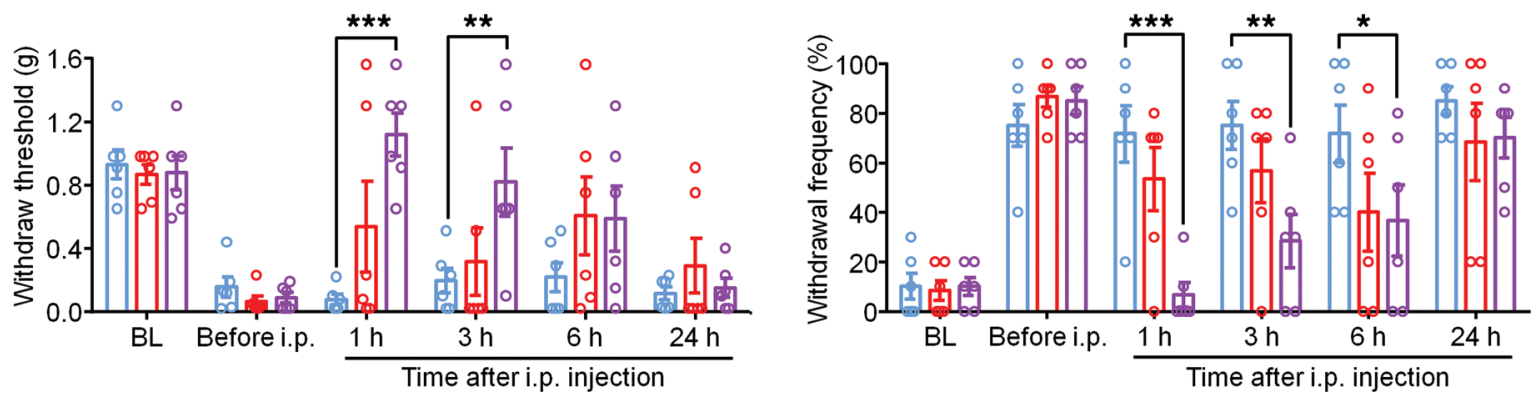
Figure 9. CCL2-CCR2 mediate PD-L1-induced osteoclast differentiation and bone cancer pain. (A-D) CCL2 levels in culture media of BMDM (A-C) and in femur BM (D), revealed by ELISA. (A) Time-dependent CCL2 release during BM cell culture. MCSF, $20 \mathrm{ng} / \mathrm{mL}$; RANKL, $40 \mathrm{ng} / \mathrm{mL}$. $n=3$ cultures. (B) Effects of PD-L1 (100 ng/mL, 1 hour) and SP600125 (20 $\mu \mathrm{M})$ on CCL2 secretion. RANKL, $20 \mathrm{ng} / \mathrm{mL} . n=4$ cultures. (C) Effects of PD-L1 (100 ng/ $\mathrm{mL}, 1$ hour) and nivolumab $(1 \mu \mathrm{g} / \mathrm{mL})$ on PD-L1 secretion. $n=3$ cultures. (D) CCL2 levels in BM lysate from naive mice and ipsilateral or contralateral side of LLC-inoculated mice on day 8. (E) Effects of CCL2 $(50 \mathrm{ng} / \mathrm{mL})$ and RS $504393(1 \mu \mathrm{g} / \mathrm{mL})$ on osteoclastogenesis in BM cells. MCSF, $20 \mathrm{ng} / \mathrm{mL}$; RANKL, $20 \mathrm{ng} / \mathrm{mL}$. Scale bar: $50 \mu \mathrm{m} . n=3$ cultures. (F) Calcium influx following CCL2 treatment $(50,100$, and $450 \mathrm{ng} / \mathrm{mL})$ in cultured DRG neurons from Advillin-GCaMP6 mice. Left: average trace ( $n=23-329$ neurons of 3-6 coverslips from 4 mice). Right: representative images of calcium responses to CCL2 and capsaicin (300 nM). Scale bar: $100 \mu \mathrm{m}$. (G and H) In situ hybridization showing Ccr2 mRNA expression in ipsilateral and contralateral DRGs 15 days after tumor inoculation. (G) Representative images for $\mathrm{Ccr} 2$ mRNA, TuJ1, and DAPI expression. Scale bar: $100 \mu \mathrm{m}$. Boxes 1 and 2 are enlarged images. Scale bar: $50 \mu \mathrm{m}$. (H) Quantification for $\mathbf{G}$ (3 sections per $\mathrm{DRG}, n=3$ male mice). (I) LLC-induced mechanical allodynia in mice treated with RS504393 (10 or $30 \mathrm{mg} / \mathrm{kg}$, i.p.) or vehicle (10\% DMSO). $n=6$ mice. Data in A-E are represented as mean \pm SEM. ${ }^{*} P<0.05$; ${ }^{* *} P<0.01$; ${ }^{* *} P<$ 0.001 , 1-way ANOVA with Bonferroni's post hoc test. 2-tailed Student's $t$ test used for $\mathbf{H}$. Repeated measures 2-way ANOVA with Bonferroni's post hoc test used for I. CAP, capsaicin.

expose the patellar ligament. Then a 25-gauge needle was inserted at the site of the intercondylar notch of the left femur into the femoral cavity, and the needle was then replaced with a $10 \mu \mathrm{L}$ microinjection syringe containing a $4 \mu \mathrm{L}$ suspension of tumor cells $\left(2 \times 10^{5}\right)$ and $2 \mu \mathrm{L}$ absorbable gelatin sponge solution for the closure of the injection site. The contents of the syringe were slowly injected into the femoral cavity (2 minutes). To prevent leakage of tumor cells outside bone cavity, the outside injection site was sealed with silicone adhesive (Kwik-Sil, World Precision Instruments). Animals with failed injection or movement dysfunction after surgery were excluded.

Drug treatment. For pretreatment, $10 \mathrm{mg} / \mathrm{kg}$ human IgG4 or nivolumab was diluted with PBS. IgG4 or nivolumab was i.v. injected via tail vein on days $0,3,7,10$, and 14 after tumor inoculation. For posttreatment, $10 \mathrm{mg} / \mathrm{kg}$ human IgG4 or nivolumab was given on day 5 after LLC inoculation and repeated on days 7, 10, 12, and 14. For treatment in naive mice, human IgG4 or nivolumab was injected (10 mg/ $\mathrm{kg}$, i.v.) on days $0,3,7,10$, and 14 . Mice were sacrificed on day 17 for tissue collection. For RS504393 treatment, 10 or 30 mg/kg RS504393 or vehicle was i.p. injected on day 11 after tumor inoculation.

Behavioral tests for pain. The following behavioral measurements were conducted blindly during the daytime ( 9 am-2 pm). Animals were habituated to the testing environment daily for at least 2 days before baseline testing. The von Frey test was used for testing mechanical allodynia. We confined mice in boxes placed on an elevated wire grid and stimulated their hind paws with a series of von Frey hairs with logarithmically increasing stiffness $(0.02-2.56 \mathrm{~g}$, Stoelting), presented perpendicularly to the central plantar surface. We determined the $50 \%$ paw withdrawal threshold by the up-down method (47). Mechanical hyperalgesia was assessed by PAM. Animals were lightly held, a special force sensor was worn on the operator's finger, and then a gradually increasing squeeze was applied across the knee joint of mice until the animal gave a response of pain (normally limb withdrawal). The maximum test duration was 5 seconds, and the peak amplifier automatically displayed, showing quantifiable force in grams. Heat sensitivity was tested using Hargreaves radiant heat apparatus (IITC Life Science). The hind paw withdrawal latency was recorded, with a cutoff of 20 seconds to prevent tissue damage (47). Cold sensitivity was measured by acetone test. Mice were placed on an elevated metal mesh floor, and a drop of acetone was applied to the plantar hind paw by syringe. The duration of time that animal lifted or licked the paw over a 90-second period was recorded.

Radiography of bone imaging. Osteolytic bone destruction was continuously assessed through radiography using MultiFocus by a Faxitron system (Faxitron Bioptics LLC). Radiographs of tumor-bearing femora were rated according to a 0 to 5 score scale as previously described (25): 0 , normal bone without signs of destruction; 1,1 to 3 radiolucent lesions indicative of bone destruction; 2, increased number of lesions (3 to 6 lesions) and loss of medullary bone; 3 , loss of medullary bone and erosion of cortical bone; 4 , full-thickness unicortical bone loss; 5 , full-thickness bicortical bone loss and displaced skeletal fracture. All radiographic image quantifications were obtained in a blinded fashion.

micro-CT. micro-CT analyses were performed on femurs from tumor-inoculated mice or naive mice before decalcification using a VivaCT 80 scanner with the $55-\mathrm{kVp}$ source (Scanco). Quantification of micro-CT data was calculated for distal femurs of WT and KO mice or IgG- and nivolumab-treated mice. Parameters included BV/TV, Conn.D, Tb.Th, Tb.Sp, and Tb.N within a region of $100 \mu \mathrm{m}$ and 200 $\mu \mathrm{m}$ proximal to the distal growth plate (49).

Bone histology. Mice were deeply anesthetized and perfused intracardially with $4 \%$ paraformaldehyde in $0.1 \mathrm{M}$ phosphate buffer. The femora were removed and post-fixed for 48 hours at $4^{\circ} \mathrm{C}$. After demineralization in EDTA (10\%) for 10 days, femur samples were dehydrated in graded concentrations of ethanol and embedded in paraffin. The serial sections $(5 \mu \mathrm{m})$ for trabecular bone were obtained from the distal femur with a microtome (Leica RM2235 Microsystems) for H\&E staining, TRAP, ALP staining, and immunohistochemistry.

In vitro osteoclast induction and TRAP staining. RAW 264.7 cells were incubated with $20 \mathrm{ng} / \mathrm{mL}$ RANKL for 6 days. Isolated BM cells were cultured in $\alpha$-MEM media containing $10 \%$ FBS and $1 \%$ antibioticantimycotic solution overnight. The suspended cells were collected and incubated with $10 \mathrm{ng} / \mathrm{mL} \mathrm{MCSF}$ for 3 days to get BMDM. The attached cells were further activated by $20 \mathrm{ng} / \mathrm{mL}$ RANKL and $20 \mathrm{ng} / \mathrm{mL}$ MCSF. For PD-1 and PD-L1 staining, RAW 264.7 cells were incubated with 40 $\mathrm{ng} / \mathrm{mL}$ RANKL, and BMDM were cocultured with $40 \mathrm{ng} / \mathrm{mL}$ RANKL and $20 \mathrm{ng} / \mathrm{mL} \mathrm{M-CSF}$. All cells were incubated on poly-D-lysine-coated (MilliporeSigma) coverslips in 24-well plates. After 6 to 7 days of incubation, cells were fixed by $4 \%$ paraformaldehyde and stained with TRAP-staining solution (TRAP Kit, MilliporeSigma, SLBW4002) for 10 to 30 minutes at $37^{\circ} \mathrm{C}$. Under a light microscope, $\mathrm{TRAP}^{+}$multinucleated cells ( $>3$ nuclei) were identified as osteoclasts, and the numbers of positive cells were counted in randomly selected visual fields ( 4 to 5 regions each well) using ImageJ software (NIH).

$\mathrm{Ca}^{2+}$ imaging in cultured mouse DRG neurons. DRGs were collected from young mice (4-6 weeks) of both sexes and incubated with collagenase $(1.25 \mathrm{mg} / \mathrm{mL}$, Roche)/dispase-II (2.4 units/mL, Roche) at $37^{\circ} \mathrm{C}$ for 90 minutes. DRG cells were mechanically dissociated with a flame-polished Pasteur pipette. Cells were plated on glass coverslips precoated with poly-D-lysine and grown in a neurobasal defined medium (with $2 \%$ B27 supplement, Invitrogen) with $5 \mu \mathrm{M}$ AraC and $5 \% \mathrm{CO}_{2}$ at $36.5^{\circ} \mathrm{C}$ for 24 hours before the experiment. $\mathrm{Ca}^{2+}$ imaging 


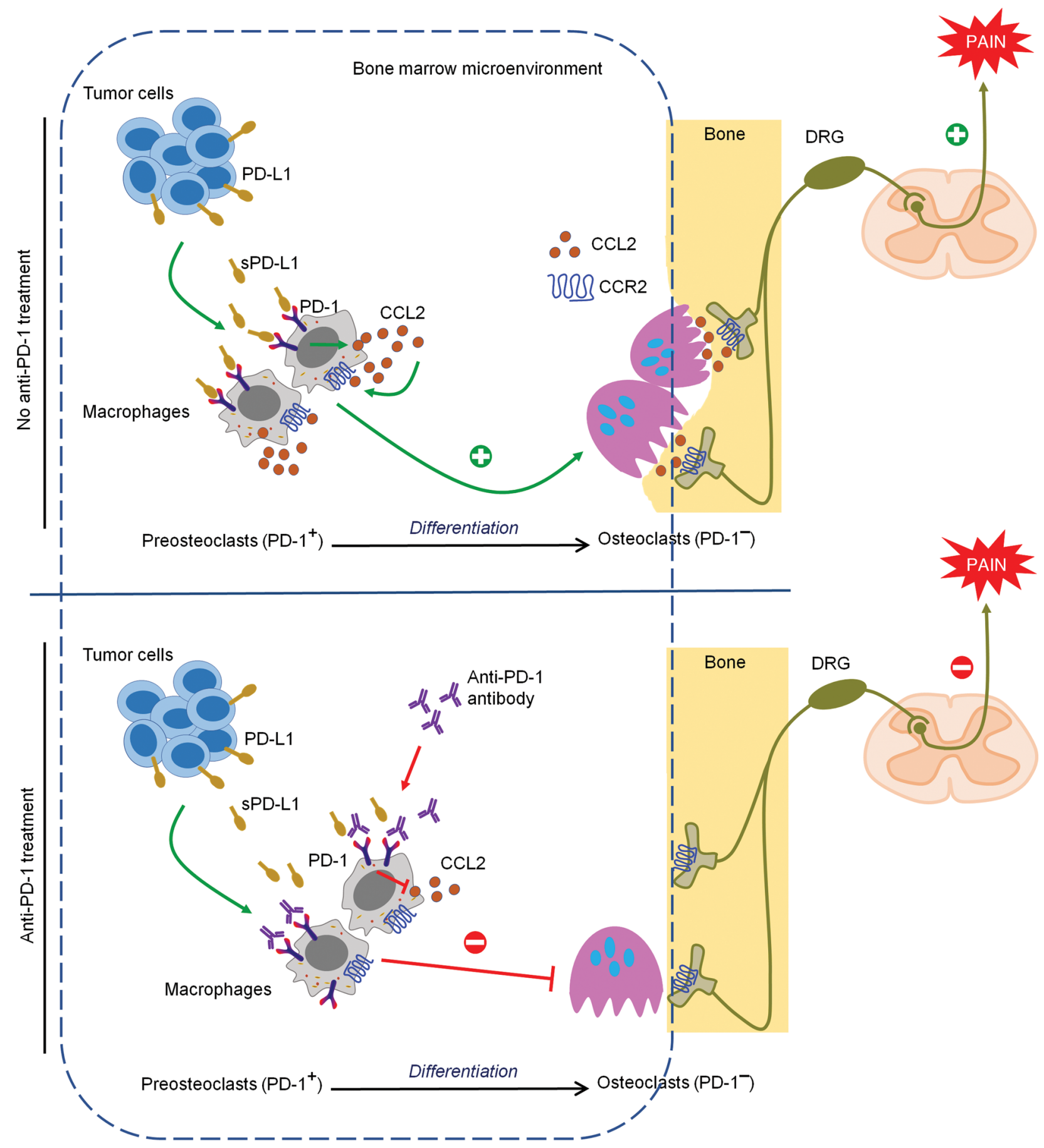

Figure 10. Schematic of PD-L1 and PD-1 axis in modulation of osteoclast differentiation, bone destruction, and bone cancer pain. In tumor-containing BM, tumor cells produce high levels of PD-L1 and further release SPD-L1. In the cancer microenvironment, PD-1 is highly expressed by preosteoclasts, including macrophages and monocytes, but not by mature osteoclasts. SPD-L1 binding PD-1 in preosteoclasts causes JNK activation and release of CCL2. CCL2 promotes the differentiation of osteoclasts. CCL2 also acts on CCR2 expressed on DRG neurons to elicit cancer pain. Furthermore, anti-PD-1 treatment with nivolumab could prevent the differentiation of preosteoclasts into osteoclasts in vitro and in vivo through the inhibition of CCL2 production and thus protect bone destruction and bone cancer pain.

was conducted in DRG neurons from Advillin-GCaMP6 mice. $\mathrm{Ca}^{2+}$ signals were captured using green emitted light in a 3-second interval. Calcium signal changes were presented as $\Delta \mathrm{F} / \mathrm{F}_{\mathrm{o}}=\left(\mathrm{F}_{\mathrm{t}}-\mathrm{F}_{\mathrm{o}}\right) / \mathrm{F}_{0}$, meaning ratio of fluorescence change $\left(\mathrm{F}_{\mathrm{t}}-\mathrm{F}_{0}\right)$ to basal value $\left(\mathrm{F}_{0}\right) . \mathrm{F}_{0}$ represents average fluorescence intensity in the baseline period. $\mathrm{F}_{\mathrm{t}}$ represents the fluorescence intensity at each indicated time point.
Flow cytometry. BM cells were enzymatically dissociated by 5 $\mathrm{mg} / \mathrm{mL}$ collagenase/dispase (Roche) in a shaking incubator for 90 minutes. For detection of cultured cells, cells were split using cold PBS/EDTA buffer. The dissociated tissues and cells were incubated in 10\% FBS-supplemented DMEM media for 1 hour for enzyme neutralization. The cells were washed following FACS analysis pro- 
tocols. All dissociated cells were blocked with $\mathrm{Fc}$ receptor staining buffer (1\% anti-mouse-CD16/CD32, 2.4 G2, 2\% FBS, 5\% NRS, and $2 \%$ NMS in HBSS; BD Biosciences) and then stained with a standard panel of antibodies. The flow cytometry events were acquired in a BD FACS Canto II flow cytometer by using BD FACS Diva 8 software (BD Biosciences). Data were analyzed using Cytobank software. Antibodies used in flow cytometry are described in Supplemental Table 2.

In vivo bioluminescence imaging. Rediject D-Luciferin Ultra was purchased from PerkinElmer (catalog 770505). Bioluminescence images of LL/2-Luc2 bearing mice were obtained with IVIS Lumina III system 15 minutes after i.p. injection of D-luciferin $(30 \mathrm{mg} / \mathrm{kg})$. The IVIS acquisition control panel was set with the following conditions for imaging: exposure time $=$ auto, binning $=$ medium, $\mathrm{F} / \mathrm{stop}=1$, emission filter $=$ open . The bioluminescence images were analyzed using Living Image software from PerkinElmer.

Statistics. Sample sizes for each experiment were based on our previous studies on such experiments $(19,25,47)$. GraphPad Prism 6.0 (GraphPad Software) was used to perform statistical analysis. All data were expressed as mean \pm SEM. Biochemical and behavioral data were analyzed using 2-tailed Student's $t$ test (2 groups), 1-way ANOVA, or 2-way ANOVA followed by post hoc Bonferroni's test. Comparison of the bone fracture ratio was completed by Fisher's exact test. $P<0.05$ was considered statistically significant.

Additional methods are listed in the Supplemental Methods.
Study approval. The present studies in animals were reviewed and approved by the IACUCs of Duke University. All animal procedures were conducted in accordance with the NIH's Guide for the Care and Use of Laboratory Animals (National Academies Press, 2011).

\section{Author contributions}

KW and RRJ designed experiments. KW, YG, SB, CRD, OC, and $\mathrm{XT}$ conducted behavioral, radiograph, Western blot, tissue culture, flow cytometry, histochemical, and calcium imaging experiments and analyzed the data. YL and AJM conducted micro-CT experiments under the supervision of $\mathrm{MJH}$. KW and RRJ wrote the paper. MJH edited the manuscript. KW and YG contributed equally to this work. KW appears first in the author list because he made the initial core findings and handled the revision of the manuscript. KW is a co-senior author, as he wrote the first draft of the manuscript.

\section{Acknowledgments}

This study was supported by Duke University funds and NIH grants R01 DE17794-13 (to RRJ) and R01AR063071 (to MJH).

Address correspondence to: Ru-Rong Ji and Kaiyuan Wang, MSRB III, 3 Genome Court, Durham, North Carolina 27710, USA. Phone: 919.684.9387 (RRJ), 984.260.2167 (KW); Email: ru-rong.ji@duke.edu, kaiyuan.wang@duke.edu.
1. Costantini M, et al. Prevalence, distress, management, and relief of pain during the last 3 months of cancer patients' life. Results of an Italian mortality follow-back survey. Ann Oncol. 2009;20(4):729-735.

2. Cleeland CS, et al. Does an oral analgesic protocol improve pain control for patients with cancer? An intergroup study coordinated by the Eastern Cooperative Oncology Group. Ann Oncol. 2005;16(6):972-980.

3. van den Beuken-van Everdingen MH, Hochstenbach LM, Joosten EA, Tjan-Heijnen VC, Janssen DJ. Update on prevalence of pain in patients with cancer: systematic review and meta-analysis. JPain Symptom Manage. 2016;51(6):1070-1090.e9.

4. Weilbaecher KN, Guise TA, McCauley LK. Cancer to bone: a fatal attraction. Nat Rev Cancer. 2011;11(6):411-425.

5. Falk S, Dickenson AH. Pain and nociception: mechanisms of cancer-induced bone pain. JClin Oncol. 2014;32(16):1647-1654.

6. Coleman RE. Clinical features of metastatic bone disease and risk of skeletal morbidity. Clin Cancer Res. 2006;12(20 Pt 2):6243s-6249s.

7. Costa L, Badia X, Chow E, Lipton A, Wardley A. Impact of skeletal complications on patients' quality of life, mobility, and functional independence. Support Care Cancer. 2008;16(8):879-889.

8. Schmidt BL. The neurobiology of cancer pain. Neuroscientist. 2014;20(5):546-562.

9. Mantyh PW, Clohisy DR, Koltzenburg M, Hunt SP. Molecular mechanisms of cancer pain. Nat Rev Cancer. 2002;2(3):201-209.

10. Ji RR, Berta T, Nedergaard M. Glia and pain: is chronic pain a gliopathy? Pain. 2013;154 Suppl 1:S10-S28.
11. Fornetti J, Welm AL, Stewart SA. Understanding the bone in cancer metastasis. J Bone Miner Res. 2018;33(12):2099-2113.

12. Schug SA, Chandrasena C. Pain management of the cancer patient. Expert Opin Pharmacother. 2015;16(1):5-15.

13. Mantyh P. Bone cancer pain: causes, consequences, and therapeutic opportunities. Pain. 2013;154 Suppl 1:S54-S62.

14. Jin HT, Ahmed R, Okazaki T. Role of PD-1 in regulating T-cell immunity. Curr Top Microbiol Immunol. 2011;350:17-37.

15. Pardoll DM. The blockade of immune checkpoints in cancer immunotherapy. Nat Rev Cancer. 2012;12(4):252-264.

16. Topalian SL, et al. Safety, activity, and immune correlates of anti-PD-1 antibody in cancer. $N$ Engl JMed. 2012;366(26):2443-2454.

17. Brahmer JR, et al. Safety and activity of antiPD-L1 antibody in patients with advanced cancer. N Engl J Med. 2012;366(26):2455-2465.

18. Ferris RL, et al. Nivolumab for recurrent squamous-cell carcinoma of the head and neck. N Engl J Med. 2016;375(19):1856-1867.

19. Chen G, et al. PD-L1 inhibits acute and chronic pain by suppressing nociceptive neuron activity via PD-1. Nat Neurosci. 2017;20(7):917-926.

20. von Moos R, Costa L, Ripamonti CI, Niepel D, Santini D. Improving quality of life in patients with advanced cancer: Targeting metastatic bone pain. Eur J Cancer. 2017;71:80-94.

21. Siva A, Xin H, Qin F, Oltean D, Bowdish KS, Kretz-Rommel A. Immune modulation by melanoma and ovarian tumor cells through expression of the immunosuppressive molecule CD200. Cancer Immunol Immunother.
2008;57(7):987-996

22. Minas K, Liversidge J. Is the CD200/CD200 receptor interaction more than just a myeloid cell inhibitory signal? Crit Rev Immunol. 2006;26(3):213-230.

23. Cui W, et al. CD200 and its receptor, CD200R, modulate bone mass via the differentiation of osteoclasts. Proc Natl Acad Sci USA. 2007;104(36):14436-14441.

24. Nagahama K, et al. The deficiency of immunoregulatory receptor $\mathrm{PD}-1$ causes mild osteopetrosis. Bone. 2004;35(5):1059-1068.

25 . Honore $\mathrm{P}$, et al. Osteoprotegerin blocks bone cancer-induced skeletal destruction, skeletal pain and pain-related neurochemical reorganization of the spinal cord. Nat Med. 2000;6(5):521-528.

26. Wang Z, et al. Anti-PD-1 treatment impairs opioid antinociception in rodents and nonhuman primates. Sci Transl Med. 2020;12(531):eaaw6471.

27. Brahmer JR, et al. Phase I study of single-agent anti-programmed death-1 (MDX-1106) in refractory solid tumors: safety, clinical activity, pharmacodynamics, and immunologic correlates. J Clin Oncol. 2010;28(19):3167-3175.

28. $\mathrm{Lin} \mathrm{H}$, et al. Host expression of PD-L1 determines efficacy of PD-L1 pathway blockade-mediated tumor regression. JClin Invest. 2018;128(2):805-815.

29. Li HY, et al. The tumor microenvironment regulates sensitivity of murine lung tumors to PD-1/ PD-L1 antibody blockade. Cancer Immunol Res. 2017;5(9):767-777.

30. Guise TA, et al. Evidence for a causal role of parathyroid hormone-related protein in the pathogenesis of human breast cancer-mediated osteolysis. J Clin Invest. 1996;98(7):1544-1549.

31. Zhao C, et al. Mice lacking the intracellular cation 
channel TRIC-B have compromised collagen production and impaired bone mineralization. Sci Signal. 2016;9(428):ra49.

32. Martín-Fernández M, et al. The usefulness of bone biomarkers for monitoring treatment disease: a comparative study in osteolytic and osteosclerotic bone metastasis models. Transl Oncol. 2017;10(2):255-261.

33. Chen G, et al. Exosomal PD-L1 contributes to immunosuppression and is associated with antiPD-1 response. Nature. 2018;560(7718):382-386.

34. Teitelbaum SL. Bone resorption by osteoclasts. Science. 2000;289(5484):1504-1508.

35. Asagiri M, Takayanagi H. The molecular understanding of osteoclast differentiation. Bone. 2007;40(2):251-264.

36. Ji RR, Gereau RW, Malcangio M, Strichartz GR. MAP kinase and pain. Brain Res Rev. 2009;60(1):135-148.

37. Gao YJ, et al. JNK-induced MCP-1 production in spinal cord astrocytes contributes to central sensitization and neuropathic pain. J Neurosci. 2009;29(13):4096-4108.

38. Abbadie C, Bhangoo S, De Koninck Y, Malcangio M, Melik-Parsadaniantz S, White FA. Chemokines and pain mechanisms. Brain Res Rev.
2009;60(1):125-134.

39. Xie RG, et al. Spinal CCL2 promotes central sensitization, long-term potentiation, and inflammatory pain via CCR2: further insights into molecular, synaptic, and cellular mechanisms. Neurosci Bull. 2018;34(1):13-21.

40. Khan UA, Hashimi SM, Bakr MM, Forwood MR, Morrison NA. CCL2 and CCR2 are essential for the formation of osteoclasts and foreign body giant cells. JCell Biochem. 2016;117(2):382-389.

41. Kim MS, Day CJ, Morrison NA. MCP-1 is induced by receptor activator of nuclear factor- $\{$ kappa $\} B$ ligand, promotes human osteoclast fusion, and rescues granulocyte macrophage colony-stimulating factor suppression of osteoclast formation. J Biol Chem. 2005;280(16):16163-16169.

42. Gao YJ, et al. Selective inhibition of JNK with a peptide inhibitor attenuates pain hypersensitivity and tumor growth in a mouse skin cancer pain model. Exp Neurol. 2009;219(1):146-155.

43. Brahmer JR, et al. Health-related quality-of-life results for pembrolizumab versus chemotherapy in advanced, PD-L1-positive NSCLC (KEYNOTE-024): a multicentre, international, randomised, open-label phase 3 trial. Lancet Oncol. 2017;18(12):1600-1609.
44. Harrington KJ, et al. Nivolumab versus standard, single-agent therapy of investigator's choice in recurrent or metastatic squamous cell carcinoma of the head and neck (CheckMate 141): health-related quality-of-life results from a randomised, phase 3 trial. Lancet Oncol. 2017;18(8):1104-1115.

45. Napolitano M, Trudu L, Bertolini F. Unexpected response to nivolumab in a "fast progressor" head and neck cancer patient. Case Rep Oncol. 2019;12(3):709-714.

46. Ansari J, Ali M, Farrag A, Ali AM, Alhamad A. Efficacy of nivolumab in a patient with metastatic renal cell carcinoma and end-stage renal disease on dialysis: case report and literature review. Case Reports Immunol. 2018;2018:1623957.

47. Chen G, Park CK, Xie RG, Ji RR. Intrathecal bone marrow stromal cells inhibit neuropathic pain via TGF- $\beta$ secretion. J Clin Invest. 2015;125(8):3226-3240.

48. Yang Y, et al. Delayed activation of spinal microglia contributes to the maintenance of bone cancer pain in female Wistar rats via $\mathrm{P} 2 \mathrm{X} 7$ receptor and IL-18. J Neurosci. 2015;35(20):7950-7963.

49. Wang C, et al. NOTCH signaling in skeletal progenitors is critical for fracture repair. JClin Invest. 2016;126(4):1471-1481. 